Full length article

\title{
Multiphoton imaging of melanoma 3D models with plasmonic nanocapsules
}

\author{
Paula Zamora-Perez ${ }^{a}$, Can Xiao a, Marcos Sanles-Sobrido ${ }^{a}$, Muriel Rovira-Esteva ${ }^{a}$, \\ José Javier Conesa $^{\mathrm{b}}$, Vladimir Mulens-Arias ${ }^{\mathrm{a}}$, Daniel Jaque ${ }^{\mathrm{c}, \mathrm{d}}$, Pilar Rivera-Gil ${ }^{\mathrm{a}, *}$
}

\author{
a Integrative Biomedical Materials and Nanomedicine Lab, Department of Experimental and Health Sciences (DCEXS), Pompeu Fabra University, PRBB, Carrer \\ Doctor Aiguader 88, 08003 Barcelona, Spain \\ ${ }^{b}$ Department of Structure of Macromolecules, Centro Nacional de Biotecnología, Consejo Superior de Investigaciones Científicas (CNB-CSIC), Cantoblanco, \\ 28049, Madrid, Spain. Mistral Beamline, Experiment Division, ALBA Synchrotron (ALBA-CELLS), Barcelona, Spain \\ ${ }^{\mathrm{c}}$ Nanomaterials for Bioimaging Group (NanoBIG), Departamento de Física de Materiales, Universidad Autónoma de Madrid, C/Francisco Tomás y Valiente 7 , \\ 28049 Madrid, Spain \\ ${ }^{\mathrm{d}}$ Nanomaterials for Bioimaging Group (NanoBIG), Instituto Ramón y Cajal de Investigación Sanitaria, Ctra de Colmenar Viejo Km 9,100, Madrid, 28034 Spain
}

\section{A R T I C L E I N F O}

\section{Article history:}

Received 19 August 2021

Revised 4 January 2022

Accepted 25 January 2022

Available online $\mathrm{xxx}$

\section{Keywords:}

Plasmonic gold biocompatible nanocapsules

Two-photon luminescence

X-ray tomography

Fluorescence lifetime

Nanoparticle cell interactions

3D tumorspheres

Melanoma cancer

Bioimaging

Reactive oxygen species (ROS)

Hypoxia

\begin{abstract}
A B S T R A C T
We report the synthesis of plasmonic nanocapsules and the cellular responses they induce in 3D melanoma models for their perspective use as a photothermal therapeutic agent. The wall of the nanocapsules is composed of polyelectrolytes. The inner part is functionalized with discrete gold nanoislands. The cavity of the nanocapsules contains a fluorescent payload to show their ability for loading a cargo. The nanocapsules exhibit simultaneous two-photon luminescent, fluorescent properties and X-ray contrasting ability. The average fluorescence lifetime $(\tau)$ of the nanocapsules measured with FLIM ( 0.3 ns) is maintained regardless of the intracellular environment, thus proving their abilities for bioimaging of models such as 3D spheroids with a complex architecture. Their multimodal imaging properties are exploited for the first time to study tumorspheres cellular responses exposed to the nanocapsules. Specifically, we studied cellular uptake, toxicity, intracellular fate, generation of reactive oxygen species, and effect on the levels of hypoxia by using multi-photon and confocal laser scanning microscopy. Because of the high $\mathrm{X}$-ray attenuation and atomic number of the gold nanostructure, we imaged the nanocapsule-cell interactions without processing the sample. We confirmed maintenance of the nanocapsules' geometry in the intracellular milieu with no impairment of the cellular ultrastructure. Furthermore, we observed the lack of cellular toxicity and no alteration in oxygen or reactive oxygen species levels. These results in 3D melanoma models contribute to the development of these nanocapsules for their exploitation in future applications as agents for imaging-guided photothermal therapy.
\end{abstract}

\section{Statement of Significance}

The novelty of the work is that our plasmonic nanocapsules are multimodal. They are responsive to $\mathrm{X}$-ray and to multiphoton and single-photon excitation. This allowed us to study their interaction with 2D and 3D cellular structures and specifically to obtain information on tumor cell parameters such as hypoxia, reactive oxygen species, and toxicity. These nanocapsules will be further validated as imagingguided photothermal probes.

(C) 2022 The Authors. Published by Elsevier Ltd on behalf of Acta Materialia Inc. This is an open access article under the CC BY-NC-ND license (http://creativecommons.org/licenses/by-nc-nd/4.0/)
Abbreviations: 2D, Bi-dimensional; 2PE, Two-photon excitation; 2PL, Twophoton luminescence; 2PM, Two-photon microscopy; 3D, Three-dimensional; AuNCs, Gold nanocapsules; CLSM, Confocal laser scanning microscopy; DMEM, Dulbecco's Modified Eagle's Medium; EDTA, Ethylenediaminetetraacetic acid; FBS, Fetal bovine serum; FLIM, Fluorescence lifetime imaging; HBSS, Hank's balanced salt so- lution; HEDFM, Hyperspectral enhanced darkfield microscopy; NIR, Near infrared; ROS, Reactive oxygen species; ROS $_{\text {cyt }}$, Cytoplasmic reactive oxygen species; ROS $_{\mathrm{DNA}}$, DNA-associated reactive oxygen species; TEM, Transmission electron microscopy.

* Corresponding author.

E-mail address: pilar.rivera@upf.edu (P. Rivera-Gil). 


\section{Introduction}

The field of cancer preclinical research has benefited from the use of three-dimensional (3D) scaffold-free tumor spheroids because of their low cost and easy culture method [1,2]. The 3D models imitate the native conformation of tissues in vivo in terms of cell heterogeneity (proliferation, hypoxia, and necrosis), cellcell and cell-matrix interactions, and the gradient of nutrients and gasses $\left(\mathrm{O}_{2}\right.$ and $\left.\mathrm{CO}_{2}\right)$ that solid tumors exhibit [1,3-6]. This gradient confers 3D spheroids intrinsic chemoresistant properties [7]. 3D spheroids have been used as in vitro systems to model in vivo hypoxic conditions. 3D spheroids are formed in the presence or absence of scaffolds. Their complexity depends on the cell type used (tumor-immune cell or tumor-vascular cell interactions). The main types include multicellular spheroids, organoids, and tumorspheres. Tumorspheres are formed under the lack of adherence and serum proteins by a single tumor cell that constantly divides until forming a sphere (round shape) or a spheroid (ellipsoidal shape) with a size $<200 \mu \mathrm{m}$ [8]. This concept is based on the anchorageindependent properties of some tumor cells that can survive as aggregates in serum-free medium after detaching and leaving the tumoral niche [9], but they also mimic the in vivo environment of avascular tumors [7].

Melanoma is a type of solid tumor that is highly heterogeneous at the molecular and structural level. Melanoma tumors are inherently resistant to conventional therapies (chemotherapy and radiotherapy), and once metastasized, they are very difficult to be clinically managed. To date, very few studies have investigated the mechanisms of drug resistance. 3D cellular models have been used in melanoma research to study the mechanisms of resistance to vemurafenib, a targeted therapeutic agent. Apart from increasing the expression of drug-resistance genes, hypoxia induces the selection of apoptosis-resistant cells and therefore is associated with melanoma metastasis and prognosis $[10,11]$. Hypoxia is related to cellular metabolism and to the generation of reactive oxygen species (ROS). ROS are chemically reactive molecules involved in cell metabolism and homeostasis; when excessively generated, ROS can induce oxidative stress, thereby promoting tumorigenesis and tumor progression [12]. Melanoma presents high levels of ROS. These are generated by different components such as melanin and other enzymes present in different cellular organelles [12]. As oxidation damages the cellular components, the subcellular localization of ROS will impact the cell differently. For instance, nuclear ROS cause DNA damage, which is more relevant than cytoplasmic ROS in promotingcell death [13]. ROS generated by the mitochondria interacts with the ROS generated in the cytoplasm [13]. Nanotoxicity can also induce unwanted oxidative stress through reactivity of nanoparticles (NPs) with the environment; however, photo-responsive NPs can be used to induce irreversible oxidative levels with antitumoral effects (e.g., photothermal and photodynamic therapy) [14].

In nanomedicine, 3D tumor spheroids are becoming a solid model to study tumor cell-NP interactions. In vitro investigation and evaluation of the performance of NPs have been conventionally conducted in bi-dimensional (2D) cellular models. However, more realistic models are required for predicting in vivo the behavior of NPs. The 3D conformation of tumor spheroids, which mimics solid tumor conformation, enables a more accurate characterization of NP interactions. This is relevant for their functional validation [15-17] since different cellular behaviors have been reported for the same type of NPs when studied in 2D versus 3D models. For example, after $24 \mathrm{~h}$, quantum dots are internalized by almost all cells in 2D culture; however, they reach 3-4 cell layers (approximately $25 \mu \mathrm{m}$ ) within a 3D spheroid [7]. Negatively charged polystyrene NPs penetrate 3D models deeper than positively charged NPs [18], whereas studies on 2D models reported better uptake of positively charged NPs than negatively charged NPs.

It is clear how clinically relevant are 3D tumor models; nevertheless, the system has some challenges. The use of 3D models implies an increased thickness as compared to that for 2D systems. Similarly to in vivo studies, this challenges the use of conventional fluorescent imaging methods based on single-photon excitation due to their limited light penetration [19-21]. Multiphoton microscopy is being increasingly implemented in clinics [22]. The field of applications for NPs responsive to two-photon excitation (2PE) include photoluminescence for bioimaging with/without heat production for photothermal therapy [23-26], ROS formation for photodynamic therapy [27], and photo-triggered drug release for chemo-photothermal therapy $[28,29]$. Two-photon luminescence (2PL) is based on the use of two photons of low energy pulsed at the femtosecond range; they are absorbed by the nanomaterial, leading to its excitation with the double of the initial energy [30]. The use of low-energy near infrared (NIR) photons improves tissue penetration (up to $1 \mathrm{~mm}$ thick $[25,31]$ ) in the socalled biological optical window where the absorption and scattering of water and tissue pigments (hemoglobin, melanin, among others) are minimal [32,33]. In contrast to wide-field or confocal laser scanning microscopy (CLSM), these two photons are only absorbed in the focal plane, which reduces cell phototoxicity, fluorophore photobleaching, or quenching, while at the same time providing higher resolution images. All this makes 2PL more suitable for 3D tumorsphere imaging.

NPs provide an alternative to traditional fluorophores for 2PL. They present drawbacks like a narrow absorption spectrum, a broad emission spectrum, and poor photostability with rapid photobleaching. NPs present broader a absorption spectra, a higher quantum yield, and improved photostability [34]. Gold (Au) NPs present tunable physicochemical properties, lack of photo-blinking, and versatility for in vivo studies [35,36]. Au clusters [37], NPs [38], nanorods [39], nanoshells [40], nanourchins [41], and nanostars [42] have been reported for 2PL. In addition, multiphoton excitation (MPE) allows to detect 2PL intensity generated by NPs and to study its decay time by using fluorescence lifetime imaging microscopy (FLIM) [43, 44, 45]. This can be used to track the NPs in biological samples, not only by visualizing them but also by monitoring if they are changing. Changes in the physicochemical parameters (e.g., agglomeration) will change their optical properties and thus their lifetime [45, 46, 47]. Imaging of colorectal carcinoma spheroids with gold NP-assisted 2PL showed that small spherical NPs diffuse easily than their rod counterparts [47]. The imaging of 2D neuroblastoma and cervical cancer cells with gold nanoclusters as imaging agents has also been reported [48]. 2PL has also been reported for ex vivo imaging of renal cell carcinoma tumor biopsies containing gold nanorods, which allows the quantification of NPs accumulated after intravenous administration and intratumor accumulation [49]. Nevertheless, the incipient nanomedical use of NP-based 2PL for imaging 3D models and for understanding of NPcell interactions require deeper studies, and other cancer types and gold-based nanosystems need to be further evaluated.

Hypoxia and ROS are two factors that play a key role in melanoma pathology [50,51] and therapeutic resistance [10,52]. These factors have been poorly investigated in 3D melanoma spheroids interacting with NPs. In the present work, we have used the 2PL of $\mathrm{Au}$ nanocapsules (AuNCs) for 3D tumor imaging. As a proof of concept for cargo encapsulation, we provide evidence for the incorporation of an organic fluorophore within the cavity. A noteworthy observation is that for other cargo, the loading methodology must be adapted to match the lyophilicity and molecular weight of the cargo. We use the responsiveness of the AuNCs to 2PL and CLSM to study the interactions with melanoma tumorspheres. Furthermore, the high atomic number and X-ray at- 
tenuation properties of gold $\left(\mathrm{Z}_{\mathrm{Au}}=79\right.$, absorption edge $\left.\mathrm{k}_{\mathrm{Au}}=81\right)$ [53] compared to other conventional contrast agents, provide the AuNCs with a good contrast capability for computed tomography or soft X-ray tomography [53]. We used 2D adherent B16 murine melanoma cells and generated scaffold-free 3D melanoma spheroids. We studied the uptake, cellular fate and resistance to intracellular degradation, cytotoxic response, hypoxia, and oxidative stress in these two melanoma models. The presence of discrete $\mathrm{Au}$ nanoislands in the wall of NCs makes them suitable for photothermal therapy of melanoma tumors, thus providing the possibility to develop imaging-guided photothermal probes.

\section{Experimental methods}

\subsection{Synthesis and characterization of AuNCS}

AuNCs were synthesized following a modified protocol [55]. First, we synthesized polystyrene NPs acting as templates for the NCs. We then adsorbed oppositely charged polyelectrolytes to wrap the templates with four monolayers. We used poly(styrene) sulfonate (PSS, $70 \mathrm{kDa} \mathrm{MW}$ ) and poly(diallyl dimethylammonium chloride) (PDDA, $100 \mathrm{kDa}$ MW). We added an additional layer of gold seeds and coated the nanosystem with a silica shell overnight. We dissolved the template with chloroform (three days) and allowed the gold seeds to grow into nanostructures localized inside the cavity of the nanocapsules. Silica-coated AuNCs were placed in Milli Q water under stirring at room temperature (RT) for $48 \mathrm{~h}$ and washed $3 \times$ with Milli Q water for silica dissolution. AuNCs loaded with rhodamine $B$ were synthesized following the same procedure as described before but using a polystyrene template conjugated with Rhodamine B. This fluorescent template was synthesized by dissolving $0.15 \mathrm{~g}$ of PVP (MW: $55,000 \mathrm{kDa}$ ) and $0.128 \mathrm{~g}$ of AIBA in $50 \mathrm{ml}$ Milli $\mathrm{Q}$ water bubbled with argan for $2 \mathrm{~h}$, with temperature increased to $70{ }^{\circ} \mathrm{C}$ (with reflux). Then, $5 \mathrm{~g}$ of styrene was added followed by the addition of $15 \mathrm{mg}$ of rhodamine B (Sigma-Aldrich) after $5 \mathrm{~min}$, leaving the polymerization reaction for $24 \mathrm{~h}$. Subsequently, the mixture was centrifuged at $9500 \mathrm{rpm}$ for $30 \mathrm{~min}$ and washed $3 \times$ with Milli Q water. All NCs were stored at $4{ }^{\circ} \mathrm{C}$ unless otherwise stated. The NCs were diluted $1000 \times$ from the stock solution with ethanol, and after sonication, $10 \mu \mathrm{L}$ of the solution was placed in a copper grid for visualization with an electron microscope (Phillips CM100). The remaining sample was used to measure the UV-vis extinction spectrum using a spectroscope (Ultrospec 2100 Pro) and/or the FL spectrum (AuNCs loaded with rhodamine B) using a fluorescence spectrophotometer (Cary Eclipse), with the sample excited at $554 \mathrm{~nm}$. The observed signal confirmed that the presence of rhodamine $b$ fluorophores inside the cavity of NCs ( $c f$. SI $\S 1$, Figure SI-3). We did not exclude the leakage of the dye during the process of core dissolution. Nevertheless, the signal was sufficient to track the NCs. Moreover, we have previously demonstrated that despite the squeezing of NCs by the cells, the cargo is not lost [56,57]. The CLSM images of AuNCs loaded with rhodamine B were obtained after the addition of one drop of the stock solution onto a glass coverslip, followed by visualization with a $100 \times$ oil immersion objective.

\subsection{Establishment and characterization of cellular models}

B16 murine melanoma adherent cells (2D model) were cultured in high glucose $(4500 \mathrm{mg} / \mathrm{L})$ Dulbecco's Modified Eagle's Medium (Gibco) supplemented with $1 \%$ penicillin-streptomycin (Hyclone) and $10 \%$ fetal bovine serum (FBS, Sigma-Aldrich). The cells were then trypsinized $2 \times$ per week using Hank's Balanced Salt Solution (HBSS, Cultek) and trypsin-EDTA $0.25 \%$ (Sigma-Aldrich). The cells were cultured at $37{ }^{\circ} \mathrm{C}$ under wet modified atmosphere with $5 \% \mathrm{CO}_{2}$. The cells were washed $3 \mathrm{x}$ with HBSS to remove the
FBS proteins, counted, and plated at the concentration of 750 cells per well in low-attachment 96-well plates (Corning). B16 murine melanoma spheroids were cultured for 5 days in the supplemented Mammocult Human Medium Kit (STEMCELL Technologies) following the manufacturer's instructions. For obtaining B16 tumor-derived cells, C57BL6 male mice between 12 and 24 weeks of age were used for the study. The syngeneic model was generated by injecting serum-free 100,000 B16 melanoma adherent cells in 1:1 basal medium-matrigel (Cultek) solution. The cells were counted using Trypan blue stain $0.4 \%$ (Sigma-Aldrich) at 1:1 ratio. After 15 days of tumor growth, the animals were euthanized; this was followed by tumor resection and necropsy of the animals. Cells explanted from the melanoma tumor were obtained after smashing a piece of fresh tissue against a $40-\mu \mathrm{m}$ cell strainer localized inside a sterile Petri dish containing B16 complete growth medium. The cell suspension was recovered, centrifuged at $1200 \mathrm{rpm}$ for $5 \mathrm{~min}$, washed with $50 \mathrm{ml}$ PBS $1 \times$, and dispersed in $2 \mathrm{ml}$ of complete growth medium for counting. A total of 300,000 cells were plated in a $25 \mathrm{~cm}^{2}$ flask (Corning). After 10 days, clones from the tumoral cells were obtained and showed morphological differences and different melanin content. The cells were trypsinized and replated for obtaining a homogenous population. Spheroids from this cell line were also obtained as reported before.

\subsection{Study of cell-NC interactions}

Fluorometric cytotoxicity study in melanoma adherent cells. Adherent B16 melanoma cells were seeded onto clear-bottom black 96-well plates at the density of 15,000 cells/ml. After incubation with different concentrations of AuNCs, 10\% resazurin solution (nonfluorescent) in complete growth medium was added to cells and incubated for $3 \mathrm{~h}$ for the reduction of the dye to resorufin (fluorescent). The resorufin signal was measured at $590 \mathrm{~nm}$ with a fluorescence spectrophotometer (Cary Eclipse). The viability was calculated after normalization for negative and positive controls of cell death (medium without AuNCs and with 10\% DMSO, respectively).

\subsection{CLSM cytotoxicity study in melanoma spheroids}

Five-day grown spheroids were placed in a 35-mm-diameter dish, treated with different concentrations of AuNCs for $24 \mathrm{~h}$, and then exposed to the fluorescent viability markers ( $\sim 10 \mathrm{~min}, \mathrm{RT})$. Propidium iodide $(10 \mu \mathrm{M})$ and CytoCalcein violet $450(5 \mu \mathrm{M})$ were used for live/dead cell FL detection. Propidium iodide is a cell death marker, and CytoCalcein violet 450 is a cell live marker. CytoCalcein violet 450 has also been reported as a detector of intracellular oxidative activity. CytoCalcein violet 450 is a fluorogenic cell permeable compound that is converted by intracellular esterases into calcein, which is a cell impermeant anion that is fluorescent, and the esterase conversion is favored by the ROS reaction. Imaging was performed with a Leica SP5 confocal microscope using a $40 \mathrm{x}$ water immersion objective and a $10-\mu \mathrm{M}$ step size for z-stack acquisition. The image of the tumorspheres was acquired with an Andor Dragonfly 500 spinning disk confocal microscope using a $40 \times$ water immersion objective and an Andor Zyla 4.2B camera. Maximum projections of the stacks were obtained with FIJI [58]. A region of interest (ROI) for each spheroid was obtained in the maximum projection, and the cell viability was calculated considering the total count per spheroid of propidium iodide and CytoCalcein violet 450 signals divided by the area of the spheroid obtained with FIJI and expressed as mean \pm SD. 


\subsection{CLSM}

Spheroids treated with different concentrations of AuNCs were imaged with a Leica SP5 confocal laser scanning microscope by using a $63 \times$ oil immersion objective and a $10-\mu \mathrm{M}$ step size for z-stack acquisition. Maximum projections of the stacks were obtained with FIJI [58]. From the maximum projection, ROIs for each spheroid were obtained. To calculate the viability, the total count for each spheroid was obtained as the count for the propidium iodide and CytoCalcein violet 450 signal divided by the area of the spheroid. Mean \pm SD values were provided in the plot. ROS and apoptosis studies were performed using 3D melanoma spheroids grown for 5 days; the spheroids were exposed for $24 \mathrm{~h}$ to $30 \mu \mathrm{g} / \mathrm{ml}$ of AuNCs, treated with $6 \mu \mathrm{M}$ CellROX green and/or CellROX deep red (Invitrogen) with $10 \mu \mathrm{M}$ apopxin (Abcam), and incubated for $1 \mathrm{~h}$. Hypoxia studies were performed using B16 melanoma spheroids grown for 5 days and treated for $24 \mathrm{~h}$ with $5 \mu \mathrm{g} / \mathrm{ml}$ in a low-attachment 96-well plate by adding LOX-1 (Organogenix) at the final concentration of $25 \mu \mathrm{M}$ for $24 \mathrm{~h}$. Internalization studies in 2D and 3D melanoma models were performed using different dyes for subcellular determination of the plasma membrane (CellMask deep red, $4000 \times$, RT, $8 \mathrm{~min}$ ), cytoplasm (CellLight ${ }^{\mathrm{TM}}$ ER-GFP, 120 $\mathrm{PPC}, 37^{\circ} \mathrm{C}, 24 \mathrm{~h}$ ), mitochondria (MitoTracker green, $2000 \times, 37{ }^{\circ} \mathrm{C}$, $30 \mathrm{~min}$ ), and lysosomes (LysoTracker green, $2000 \times, 37^{\circ} \mathrm{C}, 30 \mathrm{~min}$ ).

\subsection{Synchrotron cryo-soft X-ray microscopy}

The cryo-soft X-ray microscopy (CSXM) study was performed in B16 melanoma adherent cells seeded onto Au-EM grids coated with holey carbon (R 2/2; Quantifoil) in a manner such that 13 cells per grid's square were obtained. The cells were then incubated for $24 \mathrm{~h}$ at $37{ }^{\circ} \mathrm{C}$ in wet atmosphere and $5 \% \mathrm{CO}_{2}$ before adding the AuNCs at the concentration of $30 \mu \mathrm{g} / \mathrm{ml}$. Subsequently, the grids with control and AuNC-treated cells were incubated for 10 min with LysoTracker green $(1000 \times)$, washed with PBS $1 \times$, and vitrified by plunge-freezing them using a Leica EM GP unit. The samples were maintained in liquid nitrogen until visualization. The samples were imaged under cryo-conditions first with an epifluorescence microscope (integrated in the X-ray microscope) and then with a soft X-ray microscope (at Mistral Beamline, ALBA). Based on the FL signal, the cell candidates were selected. Next, 2D mosaics were acquired to assess sample quality, and the best candidates were acquired at $520 \mathrm{eV}$ photon energy from $-65^{\circ}$ to $65^{\circ}$ at $1^{\circ}$ interval (exposure time: $1-3 \mathrm{~s}$ ). The tilt series of images were normalized with XMIPP 3 software package [59], aligned, and reconstructed using IMOD [59,60]. Five cells per condition at the minimum were considered for the analysis. A more detailed protocol is referenced [61].

\subsection{Luminescence lifetime}

CLSM, multiphoton microscopy, and fluorescence lifetime microscopy studies were performed in a Leica SP5 confocal coupled to a femtosecond-pulsed Ti:Shappire multi-photon laser $(<80$ fs pulse) and Mai Tai DeepSee BB DS. For the experiments, cells/spheroids were placed in IBIDI plates for FLIM visualization under an inverted microscope. However, for confocal and multiphoton visualization with an upright microscope, the samples were placed in $35 \mathrm{~mm}$ diameter dishes. MPE for multiphoton microscopy and FLIM mode was performed at $\lambda_{\mathrm{ex}}=830 \mathrm{~nm}$ using a $40 \times$ water immersion objective. A step size of $10 \mu \mathrm{M}$ was applied for z-stack acquisition of 2PL from spheroids. Spheroids analyzed by FLIM were grown from passage 6 of melanoma-explanted cells. The performance of AuNCs in tissue was studied by injecting $1 \mathrm{cc}$ of $80 \mu \mathrm{g} / \mathrm{ml}$ solution in water into a $1 \mathrm{~cm}^{3}$ tissue section freshly collected from chicken muscle.

\subsection{Statistical analysis}

To confirm whether the AuNCs penetrated the spheroids and whether the penetration was dependent on AuNCs concentration, relative 2PL intensities of each $z$-stack of the melanoma spheroids (from the top to the bottom of the spheroids) were quantified and compared (Fig. 2D). Cytotoxicity of AuNCs in melanoma spheroids was determined under CLSM and expressed as mean \pm SD. The data from sample with and without AuNCs did not show a significant difference (Fig. 4). Viability test of 2D adherent B16 melanoma cells exposed to AuNCs was performed, and the results were expressed as mean \pm SD (Figure SI-16). To investigate whether AuNCs influence the production of ROS in spheroids, the mean hypoxia fluorescence signal per spheroid was determined and shown in Fig. 6C.

\section{Results and discussion}

\subsection{Synthesis and characterization of the systems: AuNCs and melanoma tumorspheres}

We first synthesized silica-coated hollow AuNCs following our reported protocols [55][93]. Next, we performed silica dissolution under strong stirring conditions for 48 h ( $c f$. SI $§ 1$, Figure SI-1 and cf. SI §2, Figure SI-7 for 3D imaging). Figs. 1A-1E (cf. SI §1, Figure SI-1) show the physicochemical characterization of the AuNCs used in this work. The Au nanostructures in the NCs' layer are visible in the TEM image (Fig. 1A). The AuNCs' extinction spectrum shows a broad localized surface plasmon resonance (LSPR) peak with the maximum intensity at $c a .570 \mathrm{~nm}$ (Fig. 1B). We used hyperspectral enhanced dark field microscopy (HEDFM) [46,62] to show the prominent light scattering properties of AuNCs in solution (bright spots) and their good colloidal dispersion and size distribution (Fig. 1C). The single and mean spectral profiles obtained by particle filtering analysis show an LSPR peak at ca. $650 \mathrm{~nm}$ (cf. SI §1, Figure SI-2).Benefiting from the hollowness of the system, we encapsulated a dye (rhodamine B Ex/Em: 543/555 nm) within the AuNCs' cavity (Fig. 1D; cf. SI $\S 1$, Figure SI-3). It is worthy to note that active ingredients/diagnostic agents can be encapsulated following the same methodology, thereby increasing the technological/clinical value of the NCs. The AuNCs are multimodal probes traceable by fluorescence and 2P microscopy. Furthermore, AuNCs show X-ray contrasting properties for soft X-ray tomography, which additionally enables evaluating NC-cell interaction in unprocessed 3D samples (Fig. 1E; $c f$. SI §3, Figure SI-13, SI-14, and SI-15).

Next, we characterized the 3D melanoma spheroids formed from adherent B16 murine melanoma cells (Fig. 1F; cf. SI §1, Figure SI-4, SI-5, and SI-6A) or from cells isolated from mice tumors (Fig. 1G; $c f$. SI $\S 1$, Figure SI-6B). B16 melanoma cells originate from immunocompetent $\mathrm{C} 57 \mathrm{Bl} / 6 \mathrm{~J}$ mice and are a realistic cellular model. We obtained 3D melanoma spheroids by removing the presence of FBS proteins from 2D adherent cells and culturing them under serum-free and ultra-low-attachment conditions for 5 days as reported elsewhere [63]. We monitored the growth of the 3D melanoma spheroids for up to 14 days. In our imaging studies, we used spheroids grown for 5 days and a surface area of $<5000$ $\mu \mathrm{m}^{2}$ ( $c f$. SI $\S 1$, Figure SI-4A and SI-4B). Cytoplasmatic ROS levels increased at the early stages of development and returned to basal levels with the time ( $c f$. SI $\S 1$, Figure SI-4C). The characterization of 2D and 3D melanoma models by CLSM showed a perinuclear organization of mitochondria and lysosomes in 3D melanoma spheroids with respect to 2D adherent cells (see Fig. 1F; $c f$. SI §1, Figure SI-5A and SI-5B). The rounding of the cells because of the non-adherent culture explains this effect. We also observed higher granularity in $3 \mathrm{D}$ melanoma spheroids than in their 2D counterparts, and there 
A

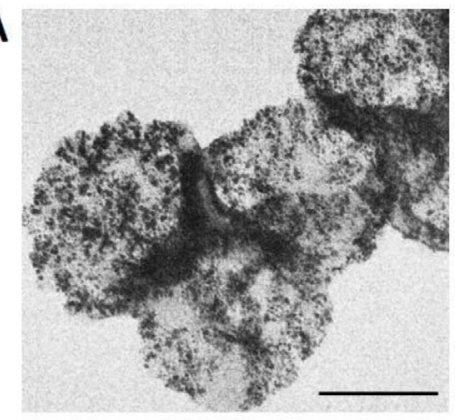

B 1

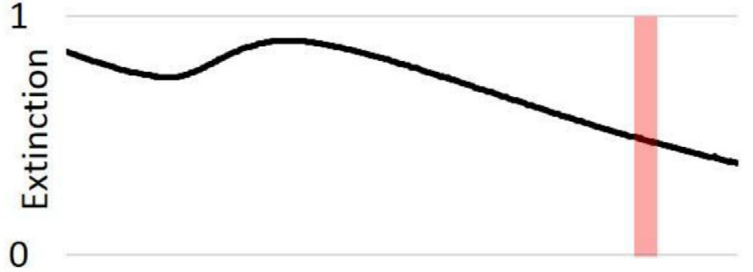

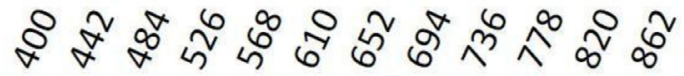 Wavelength $(\mathrm{nm})$}

C

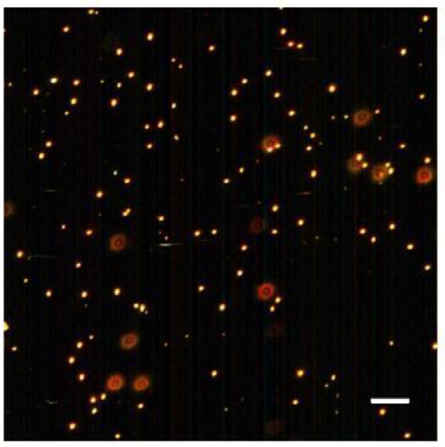

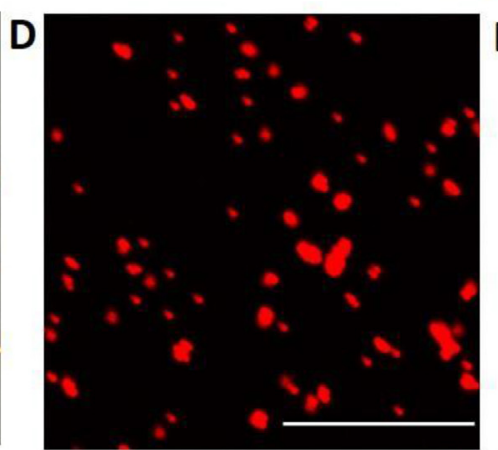

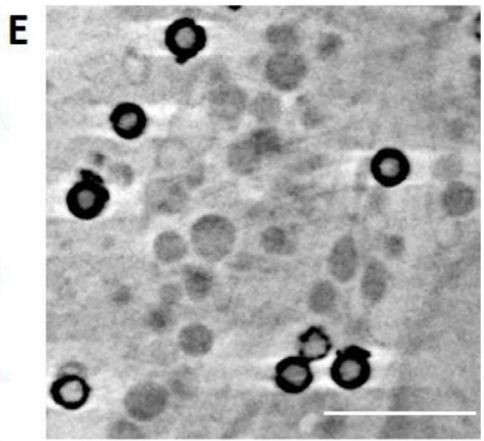

$\mathbf{F}$

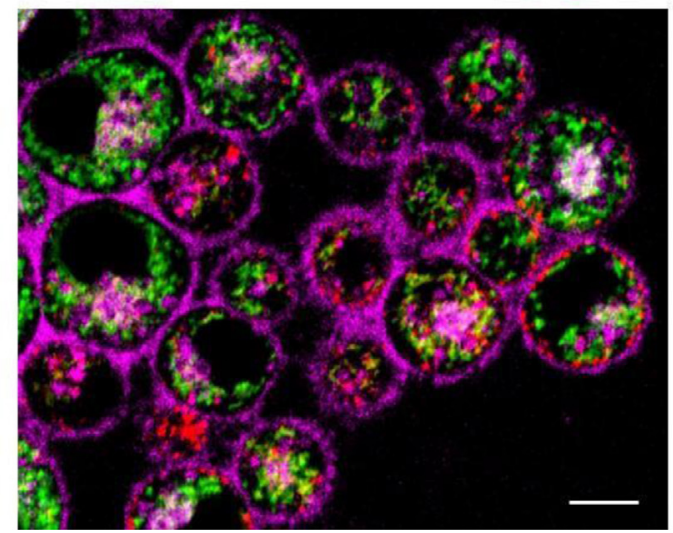

G

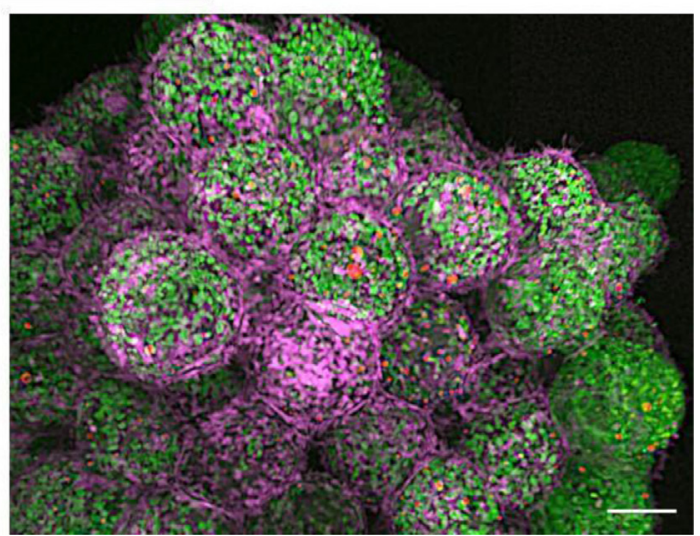

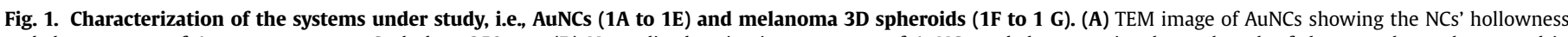

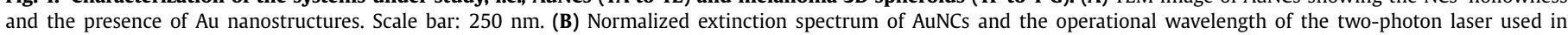

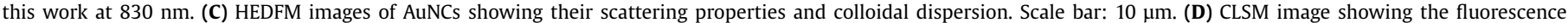

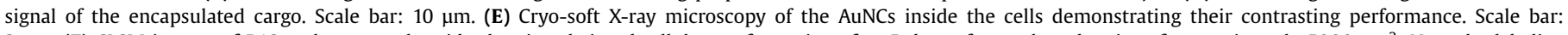

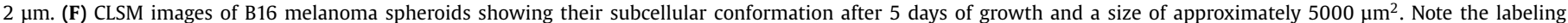

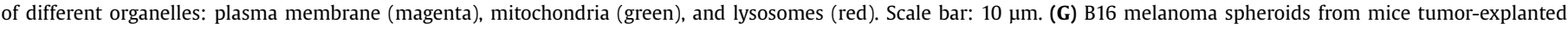

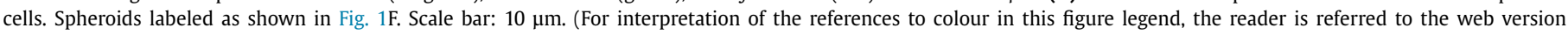
of this article.)

was no difference in size ( $c f$. SI $\S 1$, Figure SI-5A and SI-5B). Interestingly, changes in the environment (e.g., adding serum proteins and/or changing the substrate's wettability) reverted the dispersed, 3D geometry of the melanoma cells back to the adherent, 2D geometries ( $c f$. SI $\S 1$, Figure SI-5C). The reversion begins at $1 \mathrm{~h}$, and a complete loss of 3D conformation occurs after $24 \mathrm{~h}$.

\subsection{Imaging $3 D$ melanoma from in vivo models with AuNCs}

We further continued with the optical characterization of the AuNCs and studied their imaging performance in 3D models. Tumor 3D spheroids mimic in vivo conditions such as cell density and differentiated state, intercellular communication, and simulates organ-specific processes, thus constituting a validated pre-clinical model; however, their 3D architecture challenge their imaging. We wanted to confirm whether our AuNCs can be tracked within the 3D models even though they have a large thickness.
First, we characterized the AuNCs under multiphoton microscopy (MM), and we then performed concomitant MM and CLSM imaging of the melanoma 3D spheroids. Various reports have shown the excellent nonlinear optical properties of Au nanostructures compared to the best performing $2 \mathrm{P}$ absorbers, whcih depends on size and shape [48] and their use for bioimaging [6466]. Several studies have highlighted the importance of photothermal effects that may occur during MM imaging after femtosecond laser irradiation of Au NPs [67]. Because our main aim is to use these probes for imaging-guided photothermal therapy, we will require to measure the 2PA cross section of our AuNCs in future work. We measured the luminescent lifetime of the AuNCs in solution and in 3D melanoma spheroids. The luminescence lifetime $(\tau)$, the time necessary for AuNC relaxation, is an intrinsic property of the lumiphore, which is largely independent of concentration and intensity but is affected by environmental factors or factors changing the lumiphore structure [68]. By measuring the lumines- 

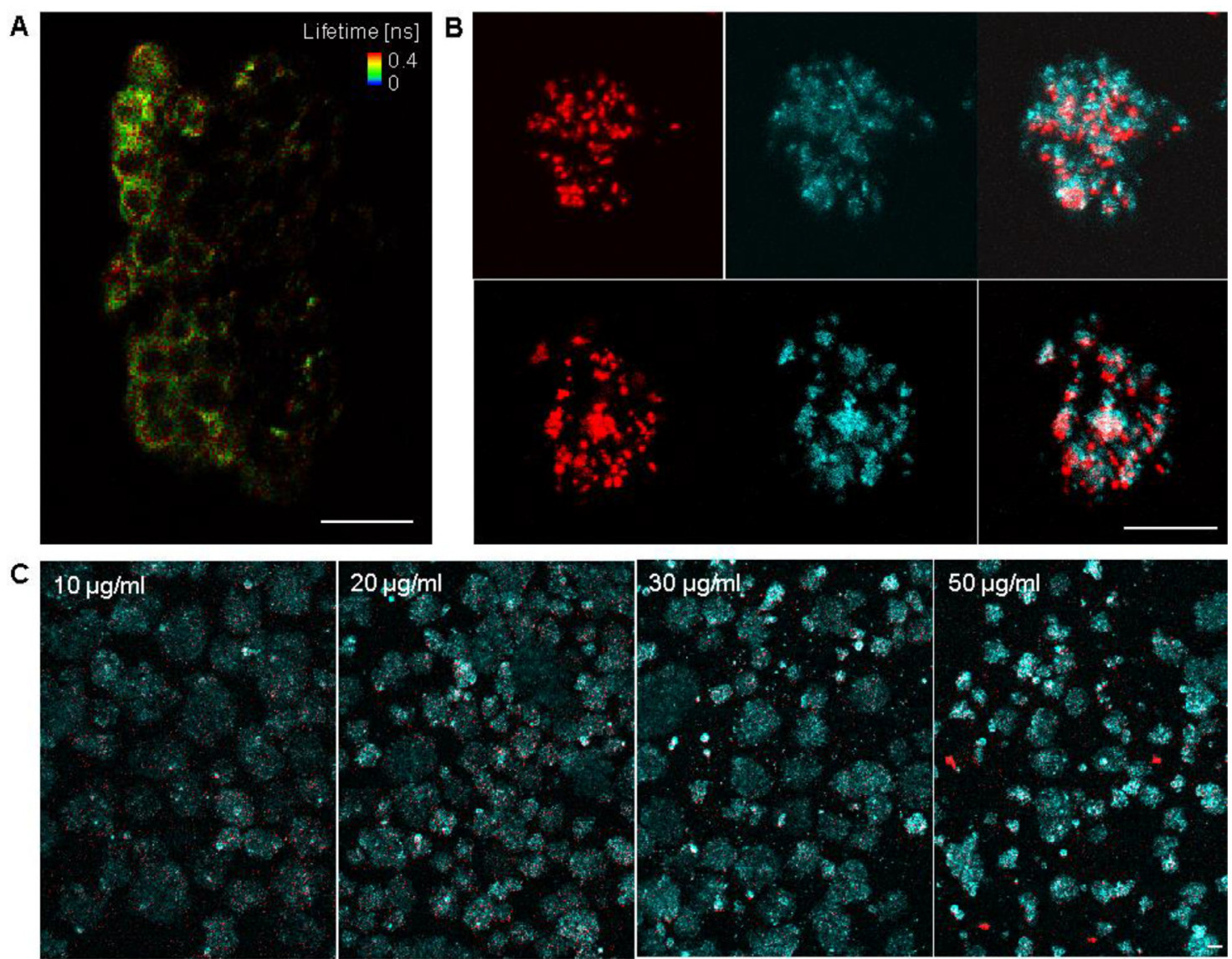

D

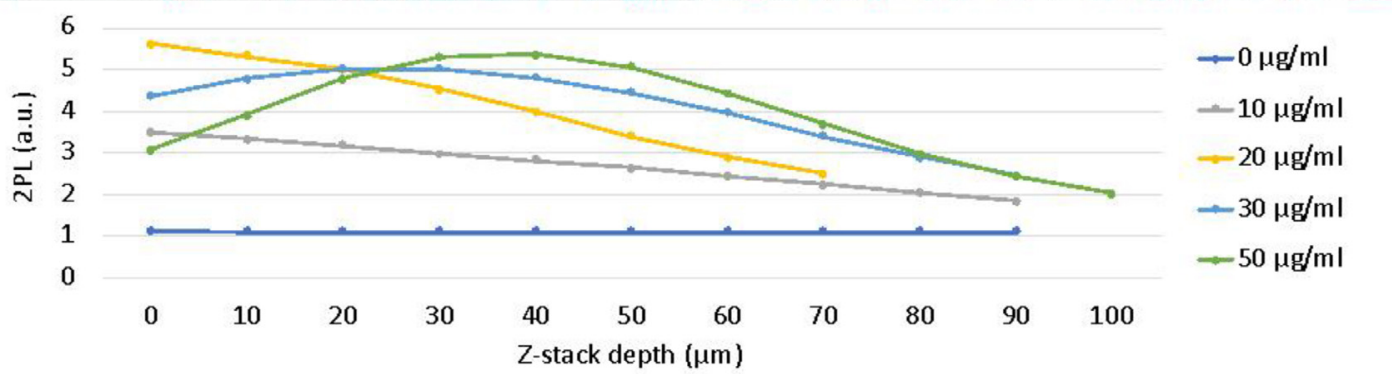

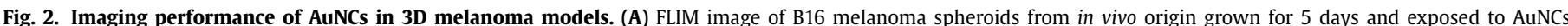

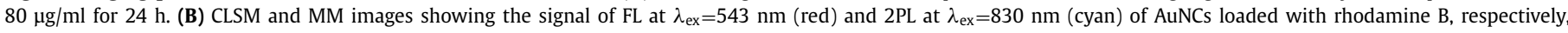

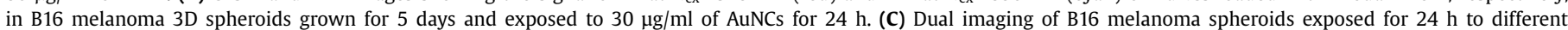

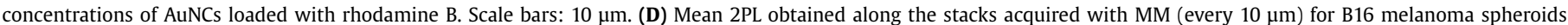
exposed to different concentrations of AuNCs. (For interpretation of the references to colour in this figure legend, the reader is referred to the web version of this article.)

cence lifetime for each pixel and generating a lifetime map, we can monitor whether changes in the physicochemical characteristics of the environment (e.g., solution $v s$. tumor) change the luminescence lifetime. Thus, we were able to assess the imaging performance of the AuNCs in the tumor. We took the 3D spheroids formed from cells isolated from the tumor and exposed them to $80 \mu \mathrm{g} / \mathrm{ml}$ AuNCs for $24 \mathrm{~h}$. After their 2PE, we measured luminescence lifetime with FLIM. AuNCs showed a $\tau<0.4$ ns and an average lifetime ( $\left.\tau_{\text {av }}\right)$ of $0.22 \mathrm{~ns}$ for the solution of AuNCs and the treated 3D melanoma spheroids (Fig. 2A; cf. SI §2, Figure SI-8). This confirmed the performance of AuNCs for MM imaging of 3D tumorspheres. Furthermore, we could image the AuNCs after 2PL in a 1-cc ex vivo tissue section of a muscle ( $c f$. SI $\S 2$, Figure SI-9), which further support their potential in vivo use as imaging probes for $2 \mathrm{PL}$.

Once we characterized in vitro (3D tumorspheres) and ex vivo the 2PL properties of AuNCs, we continued with their testing as multimodal probe for AuNC-tumorsphere interactions. We took ad- vantage of the fluorescent cargo entrapped in the AuNCs' cavity to perform dual imaging studies. The FL $\left(\lambda_{\mathrm{ex}}=543 \mathrm{~nm}\right)$ and $2 \mathrm{PL}$ $\left(\lambda_{\text {ex }}=830 \mathrm{~nm}\right)$ achieved was remarkable in both models, thus obtaining a clear identification of the interacting AuNCs (Fig. 2B; cf. SI $\S 2$, Figure SI-10). Fig. 2B shows a typical image of a 3D melanoma spheroid under concomitant MM and CLSM analysis. Fig. 2C shows how the intensity of the 2PL signal within the tumorspheres increases with the concentration of AuNCs in the range from 10 to $50 \mu \mathrm{g} / \mathrm{ml}$ ( $c f$. SI $\S 3$, Figure SI-19). We further quantified 2PL along the $\mathrm{z}$-stacks of the melanoma spheroids (obtained from the top to the bottom of the spheroids) to assess whether the penetration of AuNCs is dependent on concentration. The results show that the 2PL signal diminishes with an increasing penetration of AuNCs into the spheroids (Fig. 2D). The signal decrease exhibit a different trend depending on the concentration of AuNCs. While lower concentrations $(10-20 \mu \mathrm{g} / \mathrm{ml})$ show a linear decrease, higher concentration $(30-50 \mu \mathrm{g} / \mathrm{ml})$ show a Gaussian distribution. This result 

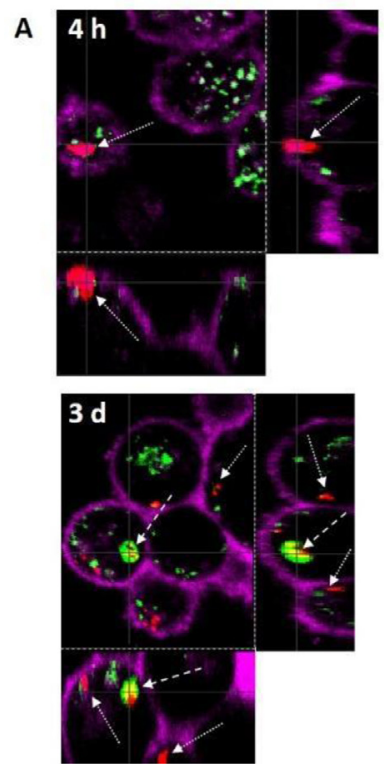
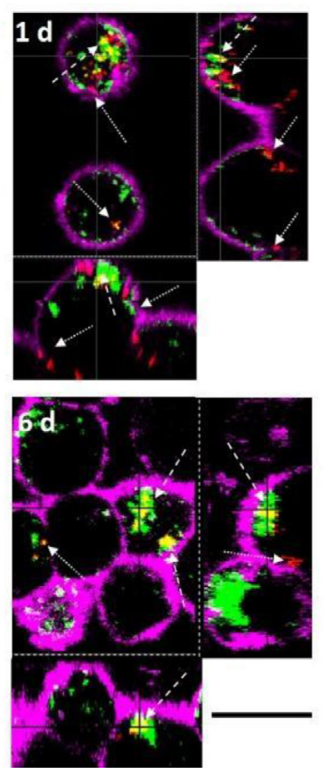

B
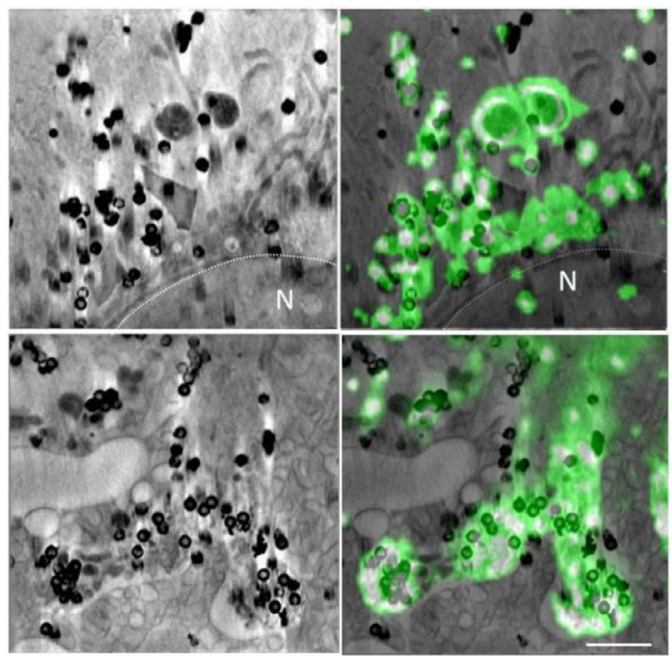

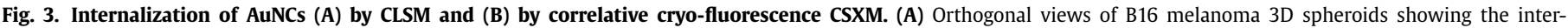

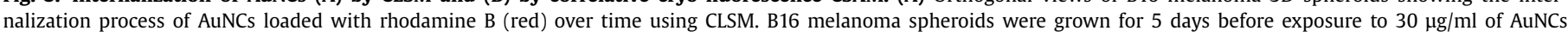

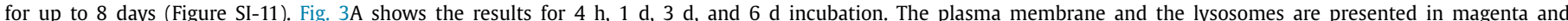

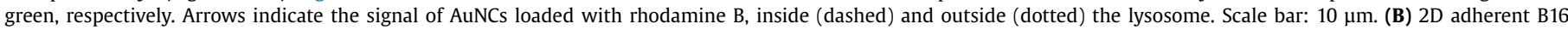

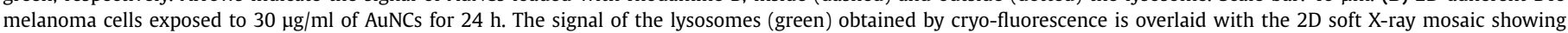

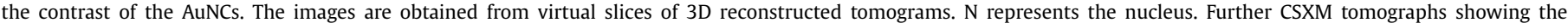

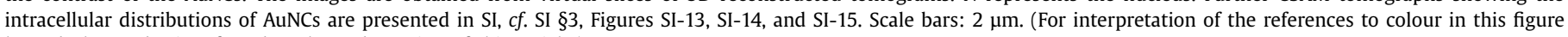
legend, the reader is referred to the web version of this article.)
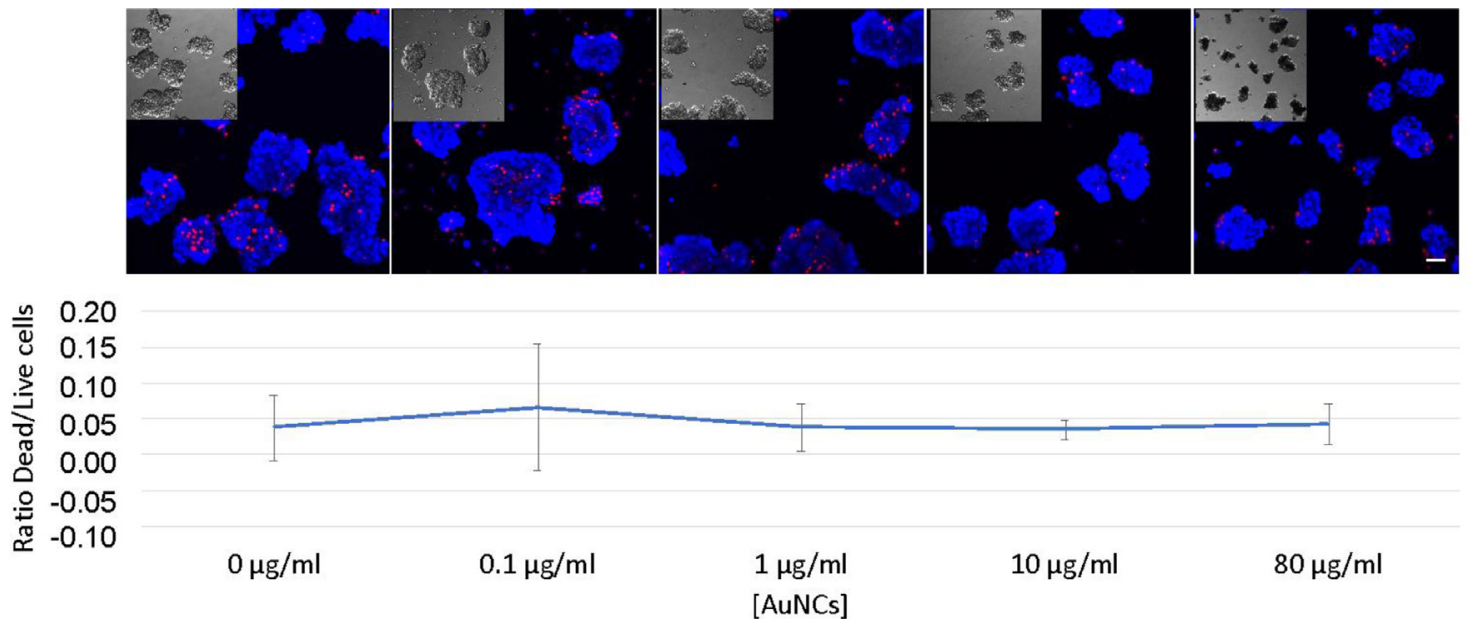

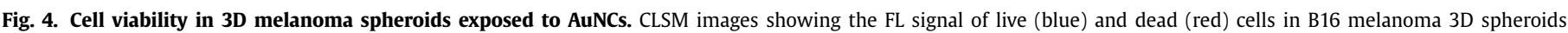

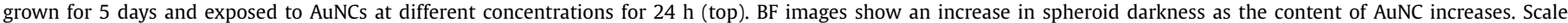

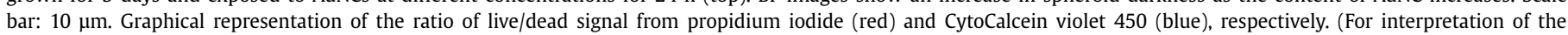
references to colour in this figure legend, the reader is referred to the web version of this article.)

indicates that the distribution of the AuNCs within the 3D spheres is not symmetric. At higher concentrations, the AuNCs penetrated deeper into the spheroids than at lower concentrations, which show a more superficial location. In the high concentration range, the bell shape signal show a wide distribution. This may indicate a high heterogeneity in the signal because of a high variability of AuNC uptake from tumorspheres to tumorspheres.

We can conclude from these experiments that AuNCs are promising candidates for use in MM for imaging multicellular 3D models. In addition to the outstanding 2PL properties of plasmonic nanomaterials, the specific geometry of the AuNCs, rationally designed to protect the functional building blocks, i.e., the plasmonic nanostructure for MM and the dye for CLSM, further contributes to their high performance. It is well known that when other types of luminescent NPs (for example, quantum dots) are dispersed in biological matrices, their quantum yield is reduced [69]. Indeed, the biological complex medium is a harsh environment that induces changes in the physicochemical properties of the NPs, which could be responsible for performance loss [62,70-72]. In plasmonic NPs, their LSPR peak responsible for their performance in the different fields of application (imaging, sensing, or therapy) is affected by the presence of biological components. Thus, an exhaustive characterization in the biological environment is crucial to validate the functionality of the nanomaterial $[62,73,74]$. We can conclude that the luminescence response of our AuNCs is maintained regardless of the harsh cellular/tissue environment. 


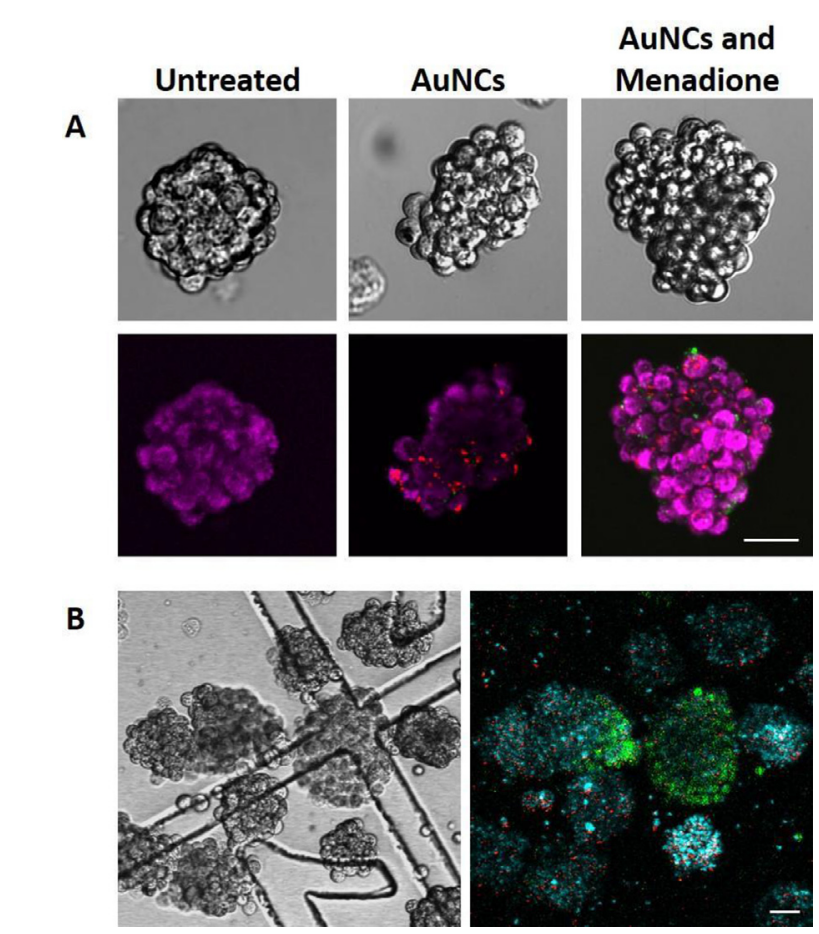

Fig. 5. Levels of ROS in the presence of AuNCs in 3D melanoma spheroids. (A) CLSM images of the melanoma spheroids not exposed and exposed to $30 \mu \mathrm{g} / \mathrm{ml}$ AuNCs (red) for $24 \mathrm{~h}$ showing the levels of ROS $_{\text {cyt }}$ (magenta) and apoptosis (green). Spheroids exposed to AuNCs show basal ROS cyt levels and those exposed to menadione $(50 \mu \mathrm{M})$ for $3 \mathrm{~h}$ as a positive control show enhanced ROS production. For further details, cf. SI $\S 3$, Figure SI-18. (B) MM and CLSM images of the melanoma spheroids exposed to $30 \mu \mathrm{g} / \mathrm{ml}$ of AuNCs loaded with rhodamine B for $24 \mathrm{~h}$ showing the signal of ROS ${ }_{\mathrm{DNA}}$ (green). 2PL of gold at $\lambda_{\mathrm{ex}}=830 \mathrm{~nm}$ (cyan) and CLSM of rhodamine at $\lambda_{\text {ex }}=543 \mathrm{~nm}$ (red). For more detailed information, $c f$. SI $\S 3$, Figure SI19. Scale bars: $10 \mu \mathrm{m}$. (For interpretation of the references to colour in this figure legend, the reader is referred to the web version of this article.)

\subsection{Internalization of AuNCs by melanoma spheroids and their viability}

Most NPs internalize into cells through endocytosis [75-77]. The endocytic pathway is interconnected with a network of acidic organelles known as lysosomes, the primary degradation system of the cell, where NPs generally accumulate $[78,79]$. The 3D conformation of the tumorspheres can affect the diffusion of the NPs [80,81]. The difficult diffusion of free or NP-loaded therapeutic compounds can be translated in vivo into poor efficacy and tumor chemoresistance. Therefore, the study of the extent to which AuNCs can diffuse through the tumorspheres is crucial. We took advantage of the luminiscent properties of AuNCs and labeled different cellular structures such as plasma membrane, mitochondria, and lysosomes to study the internalization and fate of AuNCs. Fig. 3 (cf. SI $\S 3$, Figure SI-11, SI-12, SI-13, SI-14, and SI-15) confirms the incorporation of the AuNCs within the melanoma spheroids. As commented before (Fig. 2), high concentrations (30-50 $\mu \mathrm{g} / \mathrm{ml}$ ) of AuNCs penetrated more efficiently than low concentrations (10$20 \mu \mathrm{g} / \mathrm{ml}$ ). We exposed the 3D models to $30 \mu \mathrm{g} / \mathrm{ml}$ of AuNCs and measured their uptake up to 8 days (Figs. 3A; cf. SI §3, SI-11), whereby most of them localized in the outest most layer of the tumorsphere and we did not find them in the core. As early as $4 \mathrm{~h}$ after treatment (Fig. 3A), we observed the entrapment of AuNCs by the tumorspheres. We could not confirm their lysosomal colocalization until $24 \mathrm{~h}$ post-treatment, similarly to the 2D model ( $c f$. SI §3, Figure SI-12). We did not observe a colocalization of AuNCs with the cytoplasm or with the mitochondria.
We wanted to more accurately analyze how AuNCs interact with cellular components. For this purpose, we imaged 2D adherent B16 melanoma cells with cryo-soft X-ray microscopy (CSXM) [82]. The sample requirements of this technique limit the imaging of thick samples such as 3D melanoma models. However, it enables us to study the effect of the intracellular milieu on the AuNCs and the ultrastructure of the cells interacting with the AuNCs $[83,84]$. The cells are excited in the water window $(520 \mathrm{eV})$ where carbon, the major constituent of cells, absorption is optimized. The contrast of the inorganic nature and morphology of AuNCs ease their identification and distinction from the organic cellular content. On the one side, the size of the AuNPs forming the shell have the required electron density for CSXM imaging. On the other side, the quantity of Au within the AuNCs adequately permits a certain percentage of X-ray transmittance for the appreciation of the hollow structure. With 2.5-fold better contrast per unit weight than standard agents [85], preliminary studies highlight the potential superiority of gold nanostructures. Fig. 3B shows images related to the 2D mosaic and orthoslices of 3D reconstructed tomograms containing internalized AuNCs as high-contrast (black) rings ( $c f$. SI $\S 3$, Figure SI-13, SI-14, and SI-15). In agreement with previous results ( $c f$. SI $§ 3$, Figure SI-12), CSXM confirmed the perinuclear location of AuNCs after $24 \mathrm{~h}$ of interaction with 2D adherent B16 melanoma cells ( $c f$. SI $\S 3$, Figure SI-14). CSXM images show that AuNCs are surrounded by the cellular membrane ( $c f$. SI $\S 3$, Figure SI-13), thereby confirming their location in intracellular vesicles, previously demonstrated by CLSM studies ( $c f$. SI §3, Figure SI-12C). Moreover, correlative imaging using cryo-fluorescence and CSXM localized part of the AuNCs inside the lysosomes (Fig. 4B: $c f$. SI $\S 3$, Figure SI-14 and SI-15). Under these conditions, AuNCs seem to preserve their integrity upon uptake and interaction with the intracellular milieu, even upon contact with the acidic environment of the lysosomes. This also resulted in no significant changes in the cellular structure, thus indicating the biocompatibility of AuNCs.

We next evaluated the cellular viability upon interactions with AuNCs. First, we assessed the response of 2D adherent B16 melanoma cells to AuNCs in the concentration range from 6 to $3000 \mu \mathrm{g} / \mathrm{ml}$ for 24 and $48 \mathrm{~h}$ ( $c f$. SI $\S 3$, Figures SI-16). The test measures the viability of the cells by detecting their metabolic activity. The viability of the cells is above $90 \%$ in the given concentration range. Noteworthy that our working concentration is below $80 \mu \mathrm{g} / \mathrm{ml}$ for all experiments, which is completely safe for the cells. Remarkably, at the maximum concentration $(3000 \mu \mathrm{g} / \mathrm{ml})$ where the cells show a complete black color due to the high content of AuNCs, half of the cell population was still alive after $48 \mathrm{~h}$.

We then assessed the response of the 3D melanoma spheroids exposed for $24 \mathrm{~h}$ to AuNCs in the concentration range of 0.1 to $80 \mu \mathrm{g} / \mathrm{ml}$. We stained live and dead cells and quantified the live/dead cell ratio (Fig. 4). The concentration range tested for AuNCs was limited to the maximum of $80 \mu \mathrm{g} / \mathrm{ml}$ because of the blackness of the spheroids observed in the BF image (see insets in Fig. 4). We confirm that the presence of AuNCs did not affect cell viability within the spheroid. The live/dead cell ratio for untreated and AuNC-treated melanoma spheroids was maintained along with increasing concentrations.

\subsection{Impact of the AuNCs on the ROS (ROS ${ }_{\text {cyt }}$ and ROS DNA $)$ levels of $3 \mathrm{D}$ melanoma spheroids}

The variation in ROS levels is a cellular indicator linked to growth and division but also to nanotoxicity. We elucidated the role of ROS in the 3D melanoma models upon exposure to AuNCs by analyzing the levels of ROS from different compartments: the cytoplasm (ROS cyt $_{\text {t }}$ ) and DNA-associated $\left(\operatorname{ROS}_{\mathrm{DNA}}\right)$ ROS found in the mitochondria and nucleus, respectively. If elevated, ROS cyt $_{\text {can }}$ damage proteins, mRNA, and other cytosolic molecules, whereas 

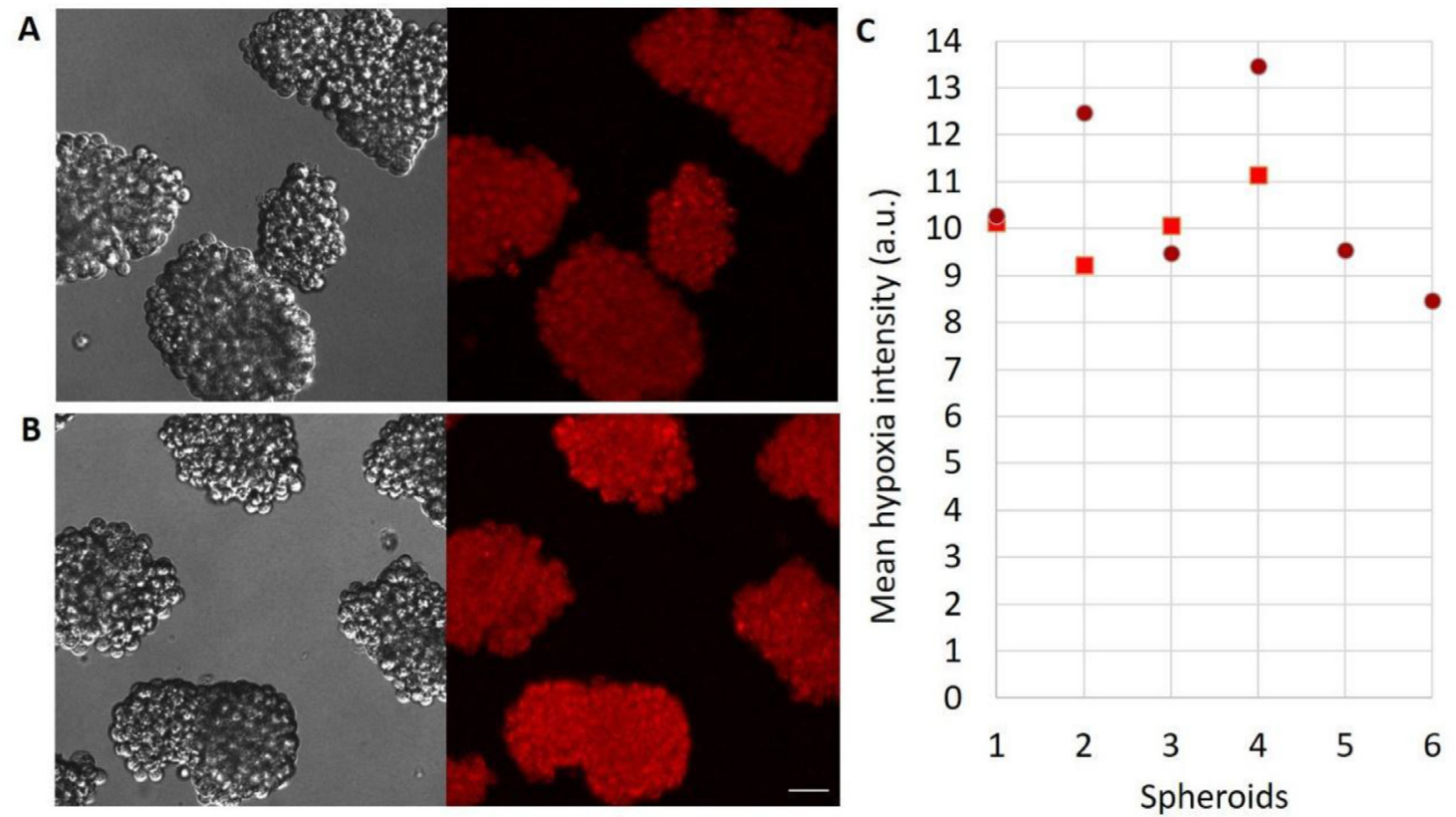

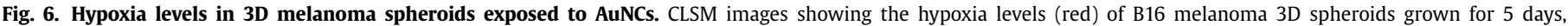

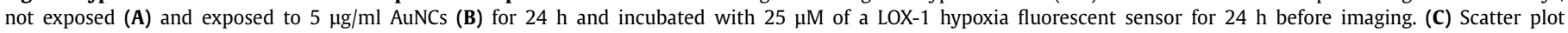

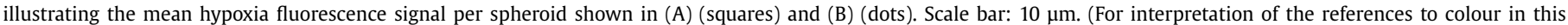
figure legend, the reader is referred to the web version of this article.)

ROS $_{\text {DNA }}$ can irreversibly affect the DNA present in the nucleus and mitochondria, leading to cell death.

First, we set up the physiological levels of $\mathrm{ROS}_{\text {cyt }}$ in $3 \mathrm{D}$ spheroids (Fig. 5A; $c f$. SI $\S 3$, Figure SI-17A) and 2D melanoma cells (cf. SI $\S 3$, Figure SI-17.B). Next, we measured the impact of AuNCs on these levels. We used a weak fluorescent probe that exhibits fluorescence in the cytoplasm upon oxidation by ROS. The basal

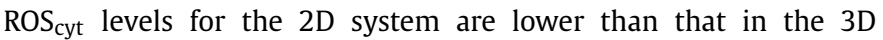
spheroids. Nevertheless, they do not induce apoptosis, and the cellular models are healthy. 3D melanoma spheroids ( $c f$. SI $\S 3$, Figure SI-17A) exhibit similar levels as previously reported in the 3D development study for 5 days of growth ( $c f$. SI $\S 1$, Figure SI-4C). As a positive control for favoring ROS production and its induced apoptosis, we exposed melanoma spheroids to menadione, a compound reported to promote oxidative stress-mediated apoptosis [86] ( $c f$. SI §3, figure SI-17.C). Melanoma spheroids exposed to menadione for $3 \mathrm{~h}$ exhibit high levels of ROS $\mathrm{S}_{\text {cyt }}$, leading to cellular apoptosis (Fig. 5A; cf. SI §3, Figure SI-17C). The presence of AuNCs do not alter the basal levels of $\operatorname{ROS}_{\text {cyt }}$ (Fig. 5A; cf. SI $\S 3$, Figure SI-18.A) and do not induce apoptosis. Spheroids not exposed to menadione and treated and not treated with AuNCs show lower ROS els and lack of apoptosis. Considering the heterogeneity in the uptake of AuNCs by each spheroid, the levels of ROS $\mathrm{S}_{\text {cyt }}$ seemed to be constant all over the spheroids, thus confirming biocompatibility of the AuNCs.

As previously mentioned in the introduction, ROS are oxygencontaining molecules generated because of activated cellular metabolism. Our previous results ( $c f$. SI $\S 3$, Figure SI-17A and 17B) show lower levels of $\mathrm{ROS}_{\text {cyt }}$ in the $2 \mathrm{D}$ than in the 3D melanoma models. Thus, we can assume that the cells in the 3D tumorspheres are metabolically more active. To confirm this, we characterized the ROS cyt levels of our 3D melanoma models upon 3D to 2D conversion. We observe a reduction in cell adherence, thus confirming that the cells in the 3D architecture are metabolically more active than the 2D cells anchored to the substrate ( $c f$. SI $\S 3$, Figure SI18B).

Our previous results also show that AuNCs are not cytotoxic, do

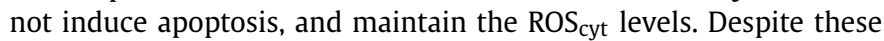
positive results, we wanted to assess the levels of ROS $_{\mathrm{DNA}}$ from mitochondrial and nuclear origin after cellular interactions with the AuNCs (Fig. 5B) to conclusively exclude ROS-mediated toxicity. Figure SI-19A shows low levels of ROS $_{\text {DNA }}$ in 3D melanoma spheroids exposed to AuNCs, which was constant throughout the concentration range of AuNCs. Benefiting from the multimodal responsiveness of AuNCs, we determined ROS $_{\text {DNA }}$ levels in the tumorspheres after AuNC internalization with 2PL and CLSM, concomitantly. Fig. 5B ( $c f$. SI §3, Figure SI-19) confirmno correlation between ROS $_{\text {DNA }}$ and the amount of internalized AuNCs, thus supporting their non-cytotoxic profile.

As it can be observed, the increase in AuNCs concentration tested in the range of $5-50 \mu \mathrm{g} / \mathrm{ml}$ in $3 \mathrm{D}$ melanoma spheroids is well reflected by the increase in 2PL. Best signal correlates with the maximum AuNCs concentration while at the same time show-

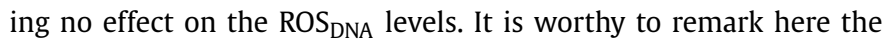
high intra-spheroid variability per AuNC condition.

Based on the results obtained in the oxidative stress studies, we can conclude that high internalization of AuNCs is associated with

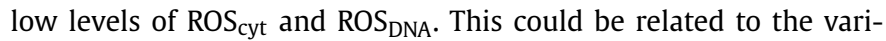
ability inherent to the studies with 3D systems or can also indicate an antioxidant-like effect provided by the AuNCs. Further investigations on this aspect are required.

In conclusion, we measured different viability parameters, (apoptosis, ROS $\mathrm{Ryt}_{\mathrm{cyt}}, \mathrm{ROS}_{\mathrm{DNA}}$, and metabolic activity) with CLSM (Fig. 5A; cf. SI §3, Figure SI-17 and 18) and 2PL (Fig. 5B; cf. SI §3, figure SI-19) and conclud that AuNCs are inert and do not alter the metabolism and viability of the tumorspheres regardless of the amount internalized. 


\subsection{Response of the 3D hypoxic tumor microenvironment model to the presence of AuNCS}

It is well known that cancer progression is regulated by tumor cells and by the microenvironment, surrounding stroma. Hypoxia is a typical feature in almost all solid tumor microenvironments. A solid tumor has different oxygenated areas [87]. Some areas are well oxygenated, while other areas lack proper oxygenation and become necrotic. Tumor cells activate specific signaling cascades with opposing effects to adapt to the hypoxic microenvironment [87]. Hypoxia is associated with a poor prognosis because it favors therapeutic resistance and malignant progression. 3D models resemble the cellular heterogeneity and oxygen gradients characteristic of in vivo tumors, which depend on their size, composition (e.g., presence of extracellular matrix) [88,89], and type of cell junctions $[90,91]$. Thus, hypoxia is reported in 3D spheroids. Hypoxia is also related to ROS in terms of cellular metabolism. By using an optical sensor whose signal is quenched in the presence of oxygen and enhanced at low oxygen conditions, we monitored hypoxia levels. As shown in Fig. 6, the hypoxia leveld of 3D melanoma spheroids is maintained regardless of presence or absence of AuNCs. This suggests that the preservation of the 3D architecture in terms of cell-cell junctions does not allow oxygen penetration. Furthermore, this result corroborates the safety profile of the AuNCs. In the case of nanotoxicity inducing cell death at the level of plasma membrane permeabilization, cell junctions might be lost, and the 3D conformation of the model would be affected. Moreover, the hypoxia levels were comparable to that of spheroids before losing the 3D conformation ( $c f$. SI $\S 3$, Figure SI20). As expected, the hypoxia levels of the tumorspheres dropped to normoxia after losing their 3D conformation. The loss was more prominent for the melanoma cells than for the control HT29 cells (cf. SI §3, Figure SI-21). This can be explained by differences in the cell-cell interactions between the two cell types. While B16 forms "grape-like" spheroids that display loose cell-cell contacts, HT29 cells are reported to generate "mass-type" spheroids, which are more tightly packed [92].

\section{Conclusion}

In conclusion, we synthesized AuNCs as a system for drug delivery because of its capability to encapsulate a cargo (modeled by a dye) and/or photothermal therapy because of their plasmonic inner wall. The AuNCs were responsive to 2PL. The average fluorescence lifetime $(\tau)$ measured with FLIM $(0.3 \mathrm{~ns})$ was maintained regardless of the intracellular environment, thus proving their abilities for bioimaging of models with a complex architecture, such as 3D spheroids and tissues. We set up a 3D melanoma tumor model and extensively characterized the interaction of AuNCs with tumor spheroids. We provide a detail picture of the cell-AuNC interactions based on the 2PL properties and the high contrast for X-ray tomography provided by the plasmonic nanostructure of the AuNCs. The uptake of AuNCs requires between 4 and $24 \mathrm{~h}$ and results in lysosomal accumulation. We measured different cellular parameters (apoptosis, $\mathrm{ROS}_{\mathrm{cyt}}$, $\mathrm{ROS}_{\mathrm{DNA}}$, and hypoxia) and conclud that AuNCs are inert and do not alter the metabolism and the viability of the tumorspheres regardless of the amount internalized. Our developed AuNCs are predictable for designing controllable nanomaterials whose properties are not erratic. The work presented here is the first step toward the development of more advanced nanosystems of interest in nanomedicine, such as imaging agents with targeting functionality and multimodal imaging properties or theranostic tools for imaging-guided therapies.

\section{Data availability statement}

The original contributions presented in the study are included in the article/Supplementary Material. Further inquiries can be directed to the corresponding author.

\section{Declaration of Competing Interest}

The authors declare that they have no known competing financial interests or personal relationships that could have appeared to influence the work reported in this paper.

\section{Acknowledgements}

Pilar Rivera Gil acknowledges the Ministry of Science, Innovation and Universities (MICINN); the AEI (AEI-PID2019106755RB-I00, RYC-2012-10059, MDM-2014-0370-04/ BES-2015075020, CTQ2013-45433-P[FEDER], MAT2016-75362-C3-2-R, AEISAF2015-73052-EXP, CEX2018-000792-M); and the AGAUR (2017 SGR 1054) for financial support. We thank the UPF/CRG Advanced Microscopy Unit (PRBB, Barcelona) for assistance in the setup of multiphoton microscopy. Work also supported by the Ministerio de Ciencia e Innovación de España (project PID2019-106211RB-I00), by the Comunidad Autónoma de Madrid (B2017/BMD-3867 RENIM$\mathrm{CM}$ ), and co-financed by the European Structural and Investment fund. Additional funding was provided by the European Union's Horizon 2020 FET Open program (Grant Agreement No. 801305, NanoTBTech), and also by COST action CA17140.

\section{Supplementary materials}

Supplementary material associated with this article can be found, in the online version, at doi:10.1016/j.actbio.2022.01.052.

\section{References}

[1] S.-M. Ong Z. Zhao, T. Arooz, D. Zhao, S. Zhang T. Du, M. Wasser, D. Van Noort, $\mathrm{H}$. Yu, Engineering a scaffold-free 3D tumor model for in vitro drug penetration studies, Biomaterials 31 (2010) 1180-1190, doi:10.1016/j.biomaterials.2009. 10.049 .

[2] M. Ravi, A. Ramesh, A. Pattabhi, Contributions of 3D Cell Cultures for Cancer Research, J. Cell. Physiol. 232 (2017) 2679-2697, doi:10.1002/jcp.25664.

[3] K. Duval, H. Grover, L.-.H. Han, Y. Mou, A.F. Pegoraro, J. Fredberg, Z. Chen, *.K. Duval, H. Grover, L.-.H. Han, Modeling Physiological Events in 2D vs. 3D Cell Culture, Physiology 32 (2017) 266-277, doi:10.1152/physiol.00036.2016.

[4] T. Rodrigues, B. Kundu, J. Silva-Correia, S.C. Kundu, J.M. Oliveira, R.L. Reis, V.M. Correlo, Emerging tumor spheroids technologies for 3D in vitro cancer modeling, Pharmacol. Ther. 184 (2018) 201-211, doi:10.1016/j.pharmthera.2017. 10.018 .

[5] M. Zanoni, F. Piccinini, C. Arienti, A. Zamagni, S. Santi, R. Polico, A. Bevilacqua, A. Tesei, 3D tumor spheroid models for in vitro therapeutic screening: a systematic approach to enhance the biological relevance of data obtained, Sci. Rep. 6 (2016) 19103, doi:10.1038/srep19103.

[6] A.S. Nunes, A.S. Barros, E.C. Costa, A.F. Moreira, I.J. Correia, 3D tumor spheroids as in vitro models to mimic in vivo human solid tumors resistance to therapeutic drugs, Biotechnol. Bioeng. 116 (2019) 206-226, doi:10.1002/bit.26845.

[7] G. Jarockyte, D. Dapkute, V. Karabanovas, J.V. Daugmaudis, F. Ivanauskas, R. Rotomskis, 3D cellular spheroids as tools for understanding carboxylated quantum dot behavior in tumors, Biochim. Biophys. Acta - Gen. Subj. 1862 (2018) 914-923, doi:10.1016/j.bbagen.2017.12.014.

[8] E.C. Costa, A.F. Moreira, D. de Melo-Diogo, V.M. Gaspar, M.P. Carvalho, I.J. Correia, 3D tumor spheroids: an overview on the tools and techniques used for their analysis, Biotechnol. Adv. 34 (2016) 1427-1441, doi:10.1016/j.biotechadv. 2016.11.002.

[9] A. Olejniczak, M. Szaryńska, Z. Kmieć, In vitro characterization of spheres derived from colorectal cancer cell lines, Int. J. Oncol. 52 (2018) 599-612, doi:10.3892/ijo.2017.4206

[10] Y. Qin, J. Roszik, C. Chattopadhyay, Y. Hashimoto, C. Liu, Z.A. Cooper, J.A. Wargo, P. Hwu, S. Ekmekcioglu, E.A. Grimm, Hypoxia-driven mechanism of vemurafenib resistance in melanoma, Mol. Cancer Ther. 15 (2016) 2442-2454, doi:10.1158/1535-7163.MCT-15-0963.

[11] R. Huber, B. Meier, A. Otsuka, G. Fenini, T. Satoh, S. Gehrke, D. Widmer M.P. Levesque, J. Mangana, K. Kerl, C. Gebhardt, H. Fujii, C. Nakashima, Y. Nonomura, K. Kabashima, R. Dummer, E. Contassot, L.E. French, Tumour hypoxia promotes melanoma growth and metastasis via High Mobility Group Box-1 and M2-like macrophages, Sci. Rep. 6 (2016) 29914, doi:10.1038/srep29914. 
[12] F. Liu-Smith, R. Dellinger, F.L. Meyskens, Updates of reactive oxygen species in melanoma etiology and progression, Arch. Biochem. Biophys. 563 (2014) 5155, doi:10.1016/j.abb.2014.04.007.

[13] L.M. Paardekooper, E. Van Vroonhoven, M. Ter Beest, G. Van Den Bogaart, Radical stress is more cytotoxic in the nucleus than in other organelles, Int. J. Mol. Sci. 20 (2019) 4147, doi:10.3390/ijms20174147.

[14] P.P. Fu, Q. Xia, H.M. Hwang, P.C. Ray, H. Yu, Mechanisms of nanotoxicity: generation of reactive oxygen species, J. Food Drug Anal. 22 (2014) 64-75, doi:10.1016/j.jfda.2014.01.005.

[15] H. Lu, M.H. Stenzel, Multicellular Tumor Spheroids (MCTS) as a 3D In Vitro Evaluation Tool of Nanoparticles, Small 14 (2018) 1702858, doi:10.1002/smll. 201702858.

[16] J.K. Wong, A. Seifalian, R. Mohseni, A.A. Hamidieh, R.E. MacLaren, N. Habib, A.M. Seifalian, Emerging In Vitro 3D Tumour Models in Nanoparticle-Based Gene and Drug Therapy, Trends Biotechnol. 36 (2018) 477-480, doi:10.1016/ j.tibtech.2018.02.002.

[17] J. Zhao, H. Lu, S. Wong, M. Lu, P. Xiao, M.H. Stenzel, Influence of nanoparticle shapes on cellular uptake of paclitaxel loaded nanoparticles in 2D and 3D cancer models, Polym. Chem. 8 (2017) 3317-3326, doi:10.1039/c7py00385d

[18] A. Tchoryk, V. Taresco, R.H. Argent, M. Ashford, P.R. Gellert, S. Stolnik, A. Grabowska, M.C. Garnett, Penetration and uptake of nanoparticles in 3D tumor spheroids, Bioconjug. Chem. 30 (2019) 1371-1384, doi:10.1021/acs. bioconjchem.9b00136.

[19] D. van den Brand, C. Veelken, L. Massuger, R. Brock, Penetration in 3D tumor spheroids and explants: adding a further dimension to the structure-activity relationship of cell-penetrating peptides, Biochim. Biophys. Acta - Biomembr. 1860 (2018) 1342-1349, doi:10.1016/j.bbamem.2018.03.007.

[20] D.L. Priwitaningrum, J.B.G. Blondé, A. Sridhar, J. van Baarlen, W.E. Hennink, G. Storm, S.Le Gac, J. Prakash, Tumor stroma-containing 3D spheroid arrays: a tool to study nanoparticle penetration, J. Control. Release. 244 (2016) 257268, doi:10.1016/j.jconrel.2016.09.004.

[21] R. Agarwal, P. Jurney, M. Raythatha, V. Singh, S.V. Sreenivasan, L. Shi, K. Roy, Effect of Shape, Size, and Aspect Ratio on Nanoparticle Penetration and Distribution inside Solid Tissues Using 3D Spheroid Models, Adv. Healthc. Mater. 4 (2015) 2269-2280, doi:10.1002/adhm.201500441.

[22] H. Lin, T. Fan, J. Sui, G. Wang, J. Chen, S. Zhuo, H. Zhang, Recent advances in multiphoton microscopy combined with nanomaterials in the field of disease evolution and clinical applications to liver cancer, Nanoscale 11 (2019) 1961919635, doi:10.1039/c9nr04902a.

[23] A.M. Larson, Multiphoton microscopy, Nat. Photonics. 5 (2011) 1, doi:10.1038/ nphoton.an.2010.2.

[24] R.M. Sandoval, B.A. Molitoris, Intravital multiphoton microscopy as a tool for studying renal physiology and pathophysiology, Methods 128 (2017) 20-32, doi:10.1016/j.ymeth.2017.07.014.

[25] P. Sarder, S. Yazdanfar, W.J. Akers, R. Tang, G.P. Sudlow, C. Egbulefu, S. Achilefu, All-near-infrared multiphoton microscopy interrogates intact tissues at deeper imaging depths than conventional single- and two-photon near-infrared excitation microscopes, J. Biomed. Opt. 18 (2013) 106012, doi:10.1117/1.jbo.18.10. 106012.

[26] E.T. Vickers, M. Garai, S. Bonabi Naghadeh, S. Lindley, J. Hibbs, Q.H. Xu, J.Z. Zhang, Two-Photon Photoluminescence and Photothermal Properties of Hollow Gold Nanospheres for Efficient Theranostic Applications, J. Phys. Chem. C. 122 (2018) 13304-13313, doi:10.1021/acs.jpcc.7b09055.

[27] Y. Shen, A.J. Shuhendler, D. Ye, J.J. Xu, H.Y. Chen, Two-photon excitation nanoparticles for photodynamic therapy, Chem. Soc. Rev. 45 (2016) 6725-6741, doi:10.1039/c6cs00442c.

[28] C.C. Huang, T.M. Liu, Controlled Au-Polymer Nanostructures for Multiphoton Imaging, Prodrug Delivery, and Chemo-Photothermal Therapy Platforms, ACS Appl. Mater. Interfaces. 7 (2015) 25259-25269, doi:10.1021/acsami.5b07110.

[29] S.M. Ardekani, A. Dehghani, M. Hassan, M. Kianinia, I. Aharonovich, V.G. Gomes, Two-photon excitation triggers combined chemo-photothermal therapy via doped carbon nanohybrid dots for effective breast cancer treatment, Chem. Eng. J. 330 (2017) 651-662, doi:10.1016/j.cej.2017.07.165.

[30] P.N. Melentiev, A.E. Afanasiev, A.A. Kuzin, A.V. Zablotskiy, V.I. Balykin, Giant enhancement of two photon induced luminescence in metal nanostructure, Opt. Express. 23 (2015) 11444, doi:10.1364/oe.23.011444.

[31] J. Liu, Two-photon microscopy in pre-clinical and clinical cancer research, Front. Optoelectron. 8 (2015) 141-151, doi:10.1007/s12200-014-0415-5

[32] C. Xu, W. Zipfel, J.B. Shear, R.M. Williams, W.W. Webb, Multiphoton fluorescence excitation: new spectral windows for biological nonlinear microscopy, Proc. Natl. Acad. Sci. U. S. A. 93 (1996) 10763-10768, doi:10.1073/pnas.93.20. 10763.

[33] V. Shanmugam, S. Selvakumar, C.S. Yeh, Near-infrared light-responsive nanomaterials in cancer therapeutics, Chem. Soc. Rev. 43 (2014) 6254-6287, doi:10. 1039/c4cs00011k.

[34] A.M. Wagner, J.M. Knipe, G. Orive, N.A. Peppas, Quantum dots in biomedical applications, Acta Biomater. 94 (2019) 44-63, doi:10.1016/j.actbio.2019.05.022.

[35] L.A. Austin, M.A. Mackey, E.C. Dreaden, M.A. El-Sayed, The optical, photothermal, and facile surface chemical properties of gold and silver nanoparticles in biodiagnostics, therapy, and drug delivery, Arch. Toxicol. 88 (2014) 1391-1417, doi:10.1007/s00204-014-1245-3.

[36] H. Yuan, C.G. Khoury, C.M. Wilson, G.A. Grant, A.J. Bennett, T. Vo-Dinh, In vivo particle tracking and photothermal ablation using plasmon-resonant gold nanostars, Nanomedicine Nanotechnology, Biol. Med. 8 (2012) 1355-1363, doi:10.1016/j.nano.2012.02.005.
[37] J. Olesiak-Banska, M. Waszkielewicz, P. Obstarczyk, M. Samoc, Two-photon absorption and photoluminescence of colloidal gold nanoparticles and nanoclusters, Chem. Soc. Rev. 48 (2019) 4087-4117, doi:10.1039/c8cs00849c.

[38] S. Suarasan, A.M. Craciun, E. Licarete, M. Focsan, K. Magyari, S. Astilean, Intracellular Dynamic Disentangling of Doxorubicin Release from Luminescent Nanogold Carriers by Fluorescence Lifetime Imaging Microscopy (FLIM) under Two-Photon Excitation, ACS Appl. Mater. Interfaces. 11 (2019) 7812-7822, doi:10.1021/acsami.8b21269.

[39] Y. Chen, Y. Zhang, D.J.S. Birch, A.S. Barnard, Creation and luminescence of size-selected gold nanorods, Nanoscale 4 (2012) 5017-5022, doi:10.1039/ c2nr30324h.

[40] M. Gordel, J. Olesiak-Banska, R. Kolkowski, K. Matczyszyn, M. Buckle, M. Samoc, Shell-thickness-dependent nonlinear optical properties of colloidal gold nanoshells, J. Mater. Chem. C. 2 (2014) 7239-7246, doi:10.1039/ c4tc01210k.

[41] E. Hutter, S. Boridy, S. Labrecque, M. Lalancette-Hébert, J. Kriz, F.M. Winnik, D. Maysinger, Microglial response to gold nanoparticles, ACS Nano 4 (2010) 2595-2606, doi:10.1021/nn901869f.

[42] Y. Liu, J.R. Ashton, E.J. Moding, H. Yuan, J.K. Register, A.M. Fales, J. Choi, M.J. Whitley, X. Zhao, Y. Qi, Y. Ma, G. Vaidyanathan, M.R. Zalutsky, D.G. Kirsch, C.T. Badea, T. Vo-Dinh, A plasmonic gold nanostar theranostic probe for in vivo tumor imaging and photothermal therapy, Theranostics 5 (2015) 946-960, doi: $10.7150 /$ thno.11974

[43] Y. Zhang, Y. Chen, J. Yu, D.J.S Birch, A surface plasmon enhanced FLIM-FRET imaging approach based on Au nanoparticles, Med. Devices Diagnostic Eng. 2 (2017) 78-82, doi:10.15761/mdde.1000119.

[44] J.S. Basuki, H.T.T. Duong, A. Macmillan, R.B. Erlich, L. Esser, M.C. Akerfeldt R.M. Whan, M. Kavallaris, C. Boyer, T.P. Davis, Using fluorescence lifetime imaging microscopy to monitor theranostic nanoparticle uptake and intracellular doxorubicin release, ACS Nano 7 (2013) 10175-10189, doi:10.1021/nn404407g.

[45] A. Boreham, R. Brodwolf, K. Walker, R. Haag, U. Alexiev, Time-Resolved Fluorescence Spectroscopy and Fluorescence Lifetime Imaging Microscopy for Characterization of Dendritic Polymer Nanoparticles and Applications in Nanomedicine, Molecules 22 (2016) 17, doi:10.3390/molecules22010017.

[46] P. Zamora-Perez, D. Tsoutsi, R. Xu, P. Rivera-Gil, Hyperspectral-enhanced dark field microscopy for single and collective nanoparticle characterization in biological environments, Materials (Basel) 11 (2018), doi:10.3390/ma11020243.

[47] T.D. Rane, A.M. Armani, Two-photon microscopy analysis of gold nanoparticle uptake in 3D cell spheroids, PLoS One 11 (2016) e0167548, doi:10.1371/journal. pone. 0167548 .

[48] J. Olesiak-Banska, M. Waszkielewicz, P. Obstarczyk, M. Samoc, Two-photon absorption and photoluminescence of colloidal gold nanoparticles and nanoclusters, Chem. Soc. Rev. 48 (2019) 4087-4117, doi:10.1039/c8cs00849c.

[49] J. Morales-Dalmau, C. Vilches, V. Sanz, I. De Miguel, V. Rodríguez-Fajardo, P. Berto, M. Martínez-Lozano, O. Casanovas, T. Durduran, R. Quidant, Quantification of gold nanoparticle accumulation in tissue by two-photon luminescence microscopy, Nanoscale 11 (2019) 11331-11339, doi:10.1039/c9nr01198f.

[50] B. Bedogni, M.B. Powell, Hypoxia, melanocytes and melanoma - Survival and tumor development in the permissive microenvironment of the skin. Pigment Cell Melanoma Res. 22 (2009) 166-174, doi:10.1111/j.1755-148X.2009.00553.x.

[51] S. Meierjohann, Oxidative stress in melanocyte senescence and melanoma transformation, Eur. J. Cell Biol. 93 (2014) 36-41, doi:10.1016/j.ejcb.2013.11.005.

[52] Y. Cheli, S. Giuliano, N. Fenouille, M. Allegra, V. Hofman, P. Hofman, P. Bahadoran, J.P. Lacour, S. Tartare-Deckert, C. Bertolotto, R. Ballotti, Hypoxia and MITF control metastatic behaviour in mouse and human melanoma cells, Oncogene 31 (2012) 2461-2470, doi:10.1038/onc.2011.425.

[53] P.A. Jackson, W.W. Nordiana Abd Rahman, C.J. Wong, T. Ackerly, M. Geso, Potential dependent superiority of gold nanoparticles in comparison to iodinated contrast agents, Eur. J. Radiol. 75 (2010) 104-109, doi:10.1016/j.ejrad.2009.03. 057.

[55] M. Sanles-sobrido, W. Exner, L. Rodrı, B. Rodrı, M.A. Correa-duarte, A.A. Ramon, L.M. Liz-marzaDesign of SERS-Encoded, , Submicron, Hollow Particles Nanoparticles, 2014, pp. 2699-2705.

[56] A.M. Javier, O. Kreft, M. Semmling, S. Kempter, A.G. Skirtach, O.T. Bruns, P. Del Pino, M.F. Bedard, J. Rädler, J. Käs, C. Plank, G.B. Sukhorukov, W.J. Parak, Uptake of colloidal polyelectrolyte-coated particles and polyelectrolyte multilayer capsules by living cells, Adv. Mater. 20 (2008) 4281-4287, doi:10.1002/ADMA. 200703190/FORMAT/PDF.

[57] P. Rivera-Gil, S. De Koker, B.G. De Geest, W.J. Parak, Intracellular processing of proteins mediated by biodegradable polyelectrolyte capsules, Nano Lett. 9 (2009) 4398-4402.

[58] J. Schindelin, I. Arganda-Carreras, E. Frise, V. Kaynig, M. Longair, T. Pietzsch, S. Preibisch, C. Rueden, S. Saalfeld, B. Schmid, J.-.Y. Tinevez, D.J. White, V. Hartenstein, K. Eliceiri, P. Tomancak, A. Cardona, Fiji: an open-source platform for biological-image analysis, Nat. Methods. 9 (2012) 676-682, doi:10. 1038/nmeth.2019.

[59] D.N. Mastronarde, S.R. Held, Automated tilt series alignment and tomographic reconstruction in IMOD, J. Struct. Biol. 197 (2017) 102-113, doi:10.1016/j.jsb. 2016.07.011.

[60] R. Carzaniga, M.C. Domart, L.M. Collinson, E. Duke, Cryo-soft X-ray tomography: a journey into the world of the native-state cell, Protoplasma 251 (2014) 449-458, doi:10.1007/s00709-013-0583-y.

[61] M. Chiappi, J.J. Conesa, E. Pereiro, C.O.S. Sorzano, M.J. Rodrguez, K. Henzler G. Schneider, F.J. Chichn, J.L. Carrascosa, Cryo-soft X-ray tomography as a quantitative three-dimensional tool to model nanoparticle: cell interaction, J. Nanobiotechnology. 14 (2016), doi:10.1186/s12951-016-0170-4. 
[62] P. Zamora Perez, B. Pélaz, D. Tsoutsi, M. G. Soliman, W. J. Parak, P. RiveraGil, Hyperspectral enhanced darkfield imaging of individual and collective hyperthermia-driven gold-copper sulfide nanoparticles, Nanoscale 13 (2021) 13256-13272, doi:10.1039/D0NR08256B.

[63] S. Sant, P.A. Johnston, The production of 3D tumor spheroids for cancer drug discovery, Drug Discov. Today Technol. 23 (2017) 27-36, doi:10.1016/j.ddtec. 2017.03.002.

[64] X. Yang, M. Yang, B. Pang, M. Vara, Y. Xia, Gold Nanomaterials at Work in Biomedicine, Chem. Rev. 115 (2015) 10410-10488, doi:10.1021/ACS.CHEMREV. 5B00193.

[65] E.C. Dreaden, A.M. Alkilany, X. Huang, C.J. Murphy, M.A. El-Sayed, The golden age: gold nanoparticles for biomedicine, Chem. Soc. Rev. 41 (2012) 2740-2779, doi:10.1039/C1CS15237H.

[66] Y. Wu, M.R.K. Ali, K. Chen, N. Fang, M.A. El-Sayed, Gold nanoparticles in biological optical imaging, Nano Today 24 (2019) 120-140, doi:10.1016/j.nantod. 2018.12.006.

[67] R. Datta, T.M. Heaster, J.T. Sharick, A.A. Gillette, M.C. Skala, Fluorescence lifetime imaging microscopy: fundamentals and advances in instrumentation, analysis, and applications, Https://Doi.Org/10.1117/1.JBO.25.7.071203. 25 (2020) 071203. https://doi.org/10.1117/1.JBO.25.7.071203.

[68] M.Y. Berezin, S. Achilefu, Fluorescence lifetime measurements and biological imaging, Chem. Rev. 110 (2010) 2641-2684, doi:10.1021/cr900343z.

[69] A.M. Wagner, J.M. Knipe, G. Orive, N.A. Peppas, Quantum dots in biomedical applications, Acta Biomater. 94 (2019) 44-63, doi:10.1016/j.actbio.2019.05.022.

[70] J. Peteiro-Cartelle, M. Rodríguez-Pedreira, F. Zhang, P.R. Gil, L.L. del Mercato, W.J. Parak, J. Peteiro-Cattelle, M. Rodriguez-Pedreira, F. Zhang, P. Rivera-Gil, L.L. del Mercato, W.J. Parak, One example on how colloidal nano- and microparticles could contribute to medicine, Nanomedicine 4 (2009) 967-979, doi:10.2217/nnm.09.84.

[71] P. Rivera-Gil, D. Jimenez de Aberasturi, V. Wulf, B. Pelaz, P. del Pino, Y. Zhao, J.M. de la Fuente, I. Ruiz de Larramendi, T. Rojo, X.J. Liang, W.J. Parak, The challenge to relate the physicochemical properties of colloidal nanoparticles to their cytotoxicity, Acc. Chem. Res. 46 (2013) 743-749, doi:10.1021/ar300039j.

[72] S. Carregal-Romero, J.M. Montenegro, W.J. Parak, P. Rivera-Gil, Subcellular carrier-based optical ion-selective nanosensors, Front. Pharmacol. (2012) 3 APR

[73] E. Petryayeva, U.J. Krull, Localized surface plasmon resonance: nanostructures, bioassays and biosensing-A review, Anal. Chim. Acta. 706 (2011) 8-24, doi:10. 1016/j.aca.2011.08.020.

[74] L.B. Sagle, L.K. Ruvuna, J.A. Ruemmele, R.P. Van Duyne, Advances in localized surface plasmon resonance spectroscopy biosensing, Nanomedicine 6 (2011) 1447-1462, doi:10.2217/nnm.11.117.

[75] P. Foroozandeh, A.A. Aziz, Insight into Cellular Uptake and Intracellular Trafficking of Nanoparticles, Nanoscale Res. Lett. 13 (2018) 1-12, doi:10.1186/ s11671-018-2728-6.

[76] I. Canton, G. Battaglia, Endocytosis at the nanoscale, Chem. Soc. Rev. 41 (2012) 2718-2739, doi:10.1039/c2cs15309b.

[77] N. Oh, J.H. Park, Endocytosis and exocytosis of nanoparticles in mammalian cells, Int. J. Nanomed. 9 (2014) 51-63, doi:10.2147/IJN.S26592.

[78] C. Schweiger, R. Hartmann, F. Zhang, W.J. Parak, T.H. Kissel, P.Rivera Gil, Quantification of the internalization patterns of superparamagnetic iron oxide nanoparticles with opposite charge, J. Nanobiotechnol. 10 (2012) 28, doi:10. 1186/1477-3155-10-28.

[79] L. Kastl, D. Sasse, V. Wulf, R. Hartmann, J. Mircheski, C. Ranke, S. CarregalRomero, J.A. Martínez-López, R. Fernández-Chacón, W.J. Parak, H.P. Elsasser, P. Rivera-Gil, Multiple internalization pathways of polyelectrolyte multilayer capsules into mammalian cells, ACS Nano 7 (2013) 6605-6618, doi:10.1021/ nn306032k.
[80] M. Durymanov, C. Kroll, A. Permyakova, J. Reineke, Role of Endocytosis in Nanoparticle Penetration of 3D Pancreatic Cancer Spheroids, Mol. Pharm. 16 (2019) 1074-1082, doi:10.1021/acs.molpharmaceut.8b01078.

[81] T.M. Achilli, S. McCalla, J. Meyer, A. Tripathi, J.R. Morgan, Multilayer spheroids to quantify drug uptake and diffusion in 3D, Mol. Pharm. 11 (2014) 2071-2081, doi: $10.1021 / \mathrm{mp} 500002 \mathrm{y}$.

[82] A. Sorrentino, J. Nicolás, R. Valcárcel, F.J. Chichón, M. Rosanes, J. Avila, A. Tkachuk, J. Irwin, S. Ferrer, E. Pereiro, MISTRAL: a transmission soft Xray microscopy beamline for cryo nano-tomography of biological samples and magnetic domains imaging, J. Synchrotron Radiat. 22 (2015) 1112-1117, doi:10.1107/S1600577515008632.

[83] M. Chiappi, J.J. Conesa, E. Pereiro, C.O.S. Sorzano, M.J. Rodrguez, K. Henzler, G. Schneider, F.J. Chichn, J.L. Carrascosa, Cryo-soft X-ray tomography as a quantitative three-dimensional tool to model nanoparticle: cell interaction, J. Nanobiotechnology. 14 (2016) 15, doi:10.1186/s12951-016-0170-4.

[84] J.J. Conesa, J. Otón, M. Chiappi, J.M. Carazo, E. Pereiro, F.J. Chichón, J.L. Carrascosa, Intracellular nanoparticles mass quantification by near-edge absorption soft X-ray nanotomography, Sci. Rep. 6 (2016) 22345, doi:10.1038/srep22354.

[85] J.M. Idée, E. Lancelot, E. Pines, C. Corot, Prophylaxis of iodinated contrast media-induced nephropathy: a pharmacological point of view, Invest. Radiol. 39 (2004) 155-170, doi:10.1097/01.RLI.0000101483.60710.2C.

[86] D.N. Criddle, S. Gillies, H.K. Baumgartner-Wilson, M. Jaffar, E.C. Chinje, S. Passmore, M. Chvanov, S. Barrow, O.V. Gerasimenko, A.V. Tepikin, R. Sutton, O.H. Petersen, Menadione-induced reactive oxygen species generation via redox cycling promotes apoptosis of murine pancreatic acinar cells, J. Biol. Chem. 281 (2006) 40485-40492, doi:10.1074/jbc.M607704200.

[87] A. Lequeux, M.Z. Noman, M. Xiao, D. Sauvage, K. Van Moer, E. Viry, I. Bocci, M. Hasmim, M. Bosseler, G. Berchem, B. Janji, Impact of hypoxic tumor microenvironment and tumor cell plasticity on the expression of immune checkpoints, Cancer Lett. 458 (2019) 13-20, doi:10.1016/j.canlet.2019.05.021.

[88] S. Riffle, R.S. Hegde, Modeling tumor cell adaptations to hypoxia in multicellular tumor spheroids, J. Exp. Clin. Cancer Res. 36 (2017), doi:10.1186/ s13046-017-0570-9.

[89] S. Däster, N. Amatruda, D. Calabrese, R. Ivanek, E. Turrini, R.A. Droeser, P. Zajac, C. Fimognari, G.C. Spagnoli, G. Iezzi, V. Mele, M.G. Muraro, Induction of hypoxia and necrosis in multicellular tumor spheroids is associated with resistance to chemotherapy treatment, Oncotarget 8 (2017) 1725-1736, doi:10. 18632/oncotarget.13857.

[90] P. Benien, A. Swami, 3D tumor models: history, advances and future perspectives, Futur. Oncol. 10 (2014) 1311-1327, doi:10.2217/fon.13.274.

[91] D.S. Reynolds, K.M. Tevis, W.A. Blessing, Y.L. Colson, M.H. Zaman, M.W. Grinstaff, Breast Cancer Spheroids Reveal a Differential Cancer Stem Cell Response to Chemotherapeutic Treatment, Sci. Rep. 7 (2017) 10382, doi:10.1038/ s41598-017-10863-4.

[92] A.C. Luca, S. Mersch, R. Deenen, S. Schmidt, I. Messner, K.-L.L. Schäfer, S.E. Baldus, W. Huckenbeck, R.P. Piekorz, W.T. Knoefel, A. Krieg, N.H. Stoecklein, Impact of the 3D Microenvironment on Phenotype, Gene Expression, and EGFR Inhibition of Colorectal Cancer Cell Lines, PLoS One 8 (2013) e59689, doi:10. 1371/journal.pone.0059689.

[93] Pilar Rivera-Gil, Carmen Vazquez-Vazquez, Vincenzo Giannini, M. Pilar Callao, Wolfgang J. Parak, Miguel A. Correa-Duarte, Ramon A. Alvarez-Puebla, Plasmonic nanoprobes for real-time optical monitoring of nitric oxide inside living cells, Angewandte Chemie International Edition 52 (51) (2013) 13694-13698 In this issue, doi:10.1002/anie.201306390. 